\title{
The $a$-theorem and the Asymptotics of 4D Quantum Field Theory
}

\author{
Markus A. Luty \\ Institut für Theoretische Physik der Universität Heidelberg \\ Heidelberg, Germany \\ Physics Department, University of California Davis \\ Davis, California 95616* \\ Joseph Polchinski \\ Kavli Institute for Theoretical Physics, University of California Santa Barbara \\ Santa Barbara, California 93106 \\ Riccardo Rattazzi \\ Institut de Théorie des Phénomènes Physiques, EPFL
}

Lausanne, Switzerland

\begin{abstract}
We study the possible IR and UV asymptotics of 4D Lorentz invariant unitary quantum field theory. Our main tool is a generalization of the Komargodski-Schwimmer proof for the $a$-theorem. We use this to rule out a large class of renormalization group flows that do not asymptote to conformal field theories in the UV and IR. We show that the only possible UV and IR asymptotics described by perturbation theory have a vanishing trace of the stress-energy tensor, and are therefore conformal. Our arguments hold even for theories with gravitational anomalies. We also give a non-perturbative argument that excludes theories with scale but not conformal invariance. This argument holds for theories in which the stress-energy tensor is sufficiently nontrivial in a technical sense that we make precise.
\end{abstract}

*Permanant address 


\section{Introduction}

In this paper we study the 4D Lorentz invariant, unitary quantum field theory in the asymptotic UV and IR limits. In every 4D theory where these asymptotics are known, they are described by a conformal field theory (CFT). For example, QCD in the chiral limit approaches a free theory of massless vector particles in the far UV, and a theory of free massless scalars (pions) in the IR. If we add quark masses, the pions get a mass and the IR theory is trivial. A more complicated example is QCD with $N_{f} \simeq \frac{11}{2} N_{c}$, which asymptotes to an interacting conformal field theory in the IR. In many cases, the UV limit is not well-defined, and we interpret these theories as effective field theories needing UV completion. Given the wealth of possible UV theories (provided by string theory, for example) it seems unlikely that we can make any definite classification of UV limits of quantum field theories. For the IR limits, it is a reasonable conjecture that all such theories are conformal field theories (or trivial).

A useful way of approaching this question is Wilson's renormalization group (RG) flow in the space of theories. The IR asymptotics is then in one-to-one correspondence with the IR behavior of the RG flow. Theories like QCD flow from one fixed point to another, as illustrated in Fig. 1a. Other more exotic possibilities are limit cycles (Fig. 1b) or ergodic behavior (Fig. 1c).

In this paper, we report on progress in ruling out $R G$ flows that do not asymptote to CFTs in the UV or IR. We demonstrate that within perturbation theory, all theories that remain perturbative in the UV or IR asymptote to a CFT. More precisely, we show that

$$
T \rightarrow 0
$$

as an operator statement, where $T=T^{\mu}{ }_{\mu}$. This means that correlation functions with one insertion of $T$ with arbitrary numbers of elementary fields asymptote to zero. In particular, this excludes perturbative $4 \mathrm{D}$ theories with scale but not conformal invariance (SFTs).

Beyond perturbation theory, we show that SFTs have a stress-energy tensor whose trace $T$ is almost trivial, in a technical sense that we make precise below. We believe that this implies that $T \equiv 0$ as an operator statement, but are unable to give a rigorous proof.

The main tool in establishing these results is a generalization of the recent proof of the $a$-theorem by Komargodski and Schwimmer (KS) [1,2]. This asserts that for 


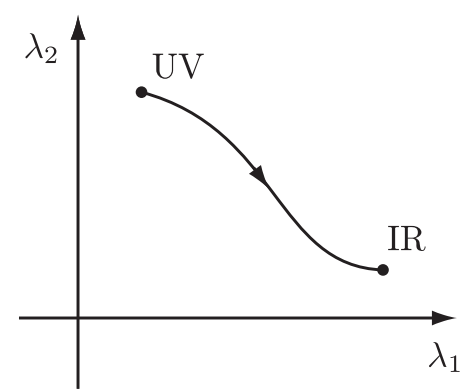

(a)

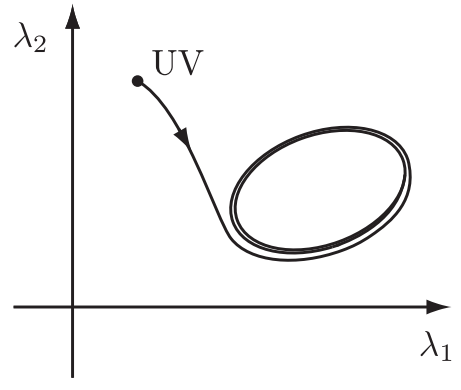

(b)

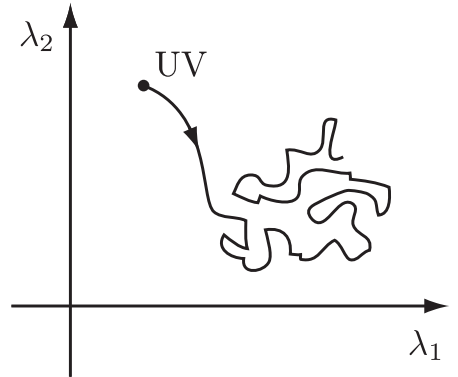

(c)

Fig. 1. A priori possible IR behavior of renormalization group flows.

theories that asymptote to CFTs both in the UV and the IR,

$$
a_{\mathrm{UV}} \geq a_{\mathrm{IR}}
$$

where $a$ is the anomaly coefficient of the CFT that describes the UV or IR limit. We will give a version of their proof that closes some potential loopholes in the original argument in Ref. [1]. We emphasize however that the key points of our proof are identical to the KS argument.

The idea is to consider the quantum field theory of interest in a conformally flat metric of the form $e^{-2 \tau(x)} \eta_{\mu \nu}$. The effective action $W[\tau]$ then defines the matrix elements of $T$ in flat spacetime. Alternatively, we can view $W[\tau]$ as the action for dilaton self-interactions obtained by integrating out the quantum field theory. This physical picture is not necessary for the argument, but it makes the arguments clearer. Following Ref. [1] we define a particular on-shell forward dilaton-dilaton scattering amplitude $A(s)$ from $W[\tau]$ that has no relevant or marginal counterterms. We write

$$
A(s)=\frac{\alpha(s) s^{2}}{f^{4}},
$$

where $s$ is the square of the center-of-mass energy, $f$ is the dilaton decay constant that counts powers of the dilaton field, and $\alpha(s)$ is a dimensionless function of $s$. The UV and IR limits of this scattering amplitude are completely determined by the " $a$ " conformal anomaly, in the sense that

$$
\alpha(s \rightarrow \infty)-\alpha(s \rightarrow 0)=-8\left(a_{\mathrm{UV}}-a_{\mathrm{IR}}\right) .
$$

This immediately relates $a_{\mathrm{UV}}-a_{\mathrm{IR}}$ to the dilaton-dilaton scattering amplitude. The left-hand side of Eq. (1.4) can be shown to be positive in unitary theories by a dispersive argument, thus proving the $a$-theorem. Our discussion pays particular attention to the convergence of the dispersion relation, which is crucial for the argument. 
To find the restrictions on general perturbative flows, we use the following logic. We define $W[\tau]$ and the dilaton-dilaton scattering amplitude as in the $a$-theorem argument. In a perturbative theory, $\alpha(s)$ is given by a power series in renormalized couplings renormalized at the scale $s$, with no counterterm. Therefore $\alpha(s)$ is bounded at all scales where perturbation theory is valid. The contour argument of Komargodski and Schwimmer is then adapted to show that the beta functions (defined as the coefficients appearing in the expansion of $T$ in local operators) must vanish in the UV and IR limits.

For non-perturbative SFTs, we use the anomalous Ward identities of scale invariance to demonstrate that $\alpha(s)$ is exactly constant. This has no imaginary part, and for unitary theories this implies that

$$
\left\langle X\left|T\left(p_{1}\right) T\left(p_{2}\right)+T\left(p_{1}+p_{2}\right)\right| 0\right\rangle \rightarrow 0
$$

for all states $|X\rangle$. In a perturbative theory, we show that this can hold only if $T \rightarrow 0$ as an operator. We cannot rigorously prove that this implies $T \equiv 0$ in general, but we give some reasons for thinking that this is the case. We test these ideas by showing that the imaginary part of the amplitude vanishes in the 4D Riva-Cardy model, a non-unitary SFT.

Non-perturbative SFTs that can be deformed to a CFT in the UV (IR) at an adjustable scale $\Lambda_{\mathrm{UV}}\left(\Lambda_{\mathrm{IR}}\right)$ are ruled out by our arguments. In such theories $\alpha(s)$ diverges at the UV (IR) as $\Lambda_{\mathrm{UV}} \rightarrow \infty\left(\Lambda_{\mathrm{IR}} \rightarrow 0\right)$, which is incompatible with the fact that the anomaly coefficient $a_{\mathrm{UV}}\left(a_{\mathrm{IR}}\right)$ of the UV (IR) CFT is finite (see Eq. (1.4)). This gives additional reasons for thinking that SFTs are impossible in general.

The arguments we use depend heavily on the consistency of the theory in a background metric. Nonetheless, we show that the arguments hold even for theories with gravitational anomalies because the anomaly does not depend on the dilaton mode. We do not consider theories that do not have a conserved stress-energy tensor, which may for example emerge as low-energy effective theories e.g. from lattice models.

This paper is organized as follows. In $\S 2$ we present the proof of the $a$-theorem. In $\S 3$ we derive the restrictions on the renormalization group flows of perturbative theories. In $\S 4$ we discuss possible non-perturbative theories with scale but not conformal invariance, and we summarize in $\S 5$. Some technical details of scale without conformal invariance in perturbation theory in an appendix.

An explicit example of a perturbative SFT was recently proposed by Fortin, Grinstein, and Stergiou in Ref. [3]. However, it has now been recognized that this theory is exactly conformal [4]. Our argument does not exclude SFTs in $4+\epsilon$ dimensions, possible examples of which were previously presented by the same authors [5]. We 
are grateful to the authors of these papers for extensive discussions of the previous version of our work, which have led us to a much clearer presentation. In the previous version we identified the dilaton scattering amplitude with the running coefficient of the WZ term. This is incorrect [6], but this identification does not actually enter into our argument — see the discussion of Eq. (3.21). These discussions have also helped us to understand the classic papers Refs. [7, 8, 9], enabling us to get a better understanding of the perturbative result, as well as providing an alternative derivation of our results for the perturbative case.

\section{The $a$-Theorem}

In this section we will present a proof of the $a$-theorem, filling in some details in the argument of Refs. [1,2]. Several of the steps of the proof will be used in the generalizations that follow.

\subsection{The Dilaton as External Field}

We introduce the dilaton as the conformal mode of the metric $g_{\mu \nu}$ by considering the theory in the background metric

$$
\hat{g}_{\mu \nu}=e^{-2 \tau} g_{\mu \nu}
$$

We will eventually take $g_{\mu \nu}=\eta_{\mu \nu}$, but we keep it general to make the general covariance of our results explicit.

We consider a UV CFT deformed by relevant operators, generating a nontrivial $\mathrm{RG}$ flow in the IR. (We will not discuss flows induced by turning on moduli fields, e.g. in supersymmetric theories.) The case of marginally relevant operators generating logarithmic flows will also be included below. The action is then

$$
S=S_{\mathrm{UV}}+\int d^{4} x \sqrt{-\hat{g}} \sum_{i} c_{i} m^{4-\Delta_{i}} \hat{\mathcal{O}}_{i}
$$

where $S_{\mathrm{UV}}$ is the action of the UV CFT, $\hat{\mathcal{O}}_{i}$ are relevant primary operators with dimension $\Delta_{i}<4, m$ is the mass scale associated with the flow, and $c_{i}$ are dimensionless coefficients. (Descendant operators are total derivatives, and therefore do not deform the theory.) We do not include irrelevant operators in the action because this would change the UV behavior of the theory. In other words, we are softly breaking the conformal symmetry of the UV CFT. In the presence of the perturbation above, the UV behavior of the theory is governed by the UV CFT, but there is a nontrivial flow 
at the scale $m$ where conformal invariance is explicitly broken. In this section we will assume that in the IR the theory flows to a different IR CFT; we will consider more general IR behavior in the following sections.

Conformal transformations are the subgroup of Weyl $\times$ diffeormorphisms that leave the flat space metric invariant, where Weyl transformations are defined by ${ }^{1}$

$$
\hat{g}_{\mu \nu} \mapsto e^{2 \sigma} \hat{g}_{\mu \nu}, \quad \hat{\mathcal{O}} \mapsto e^{-\sigma \Delta} \hat{\mathcal{O}}
$$

with $\sigma$ a general function of $x$.

We now consider the theory in the background metric $\hat{g}_{\mu \nu}=e^{-2 \tau} g_{\mu \nu}$ introduced above. The field $\tau$ is a redundant variable, since it can be eliminated by the trivial gauge invariance

$$
\tau \mapsto \tau+\alpha, \quad g_{\mu \nu} \mapsto e^{2 \alpha} g_{\mu \nu}
$$

This transformation acts trivially on the CFT fields. The existence of the field $\tau$ allows us to define Weyl transformations Eq. (2.3) as acting on the dilaton and leaving $g_{\mu \nu}$ invariant:

$$
\tau \mapsto \tau-\sigma, \quad g_{\mu \nu} \mapsto g_{\mu \nu}, \quad \hat{\mathcal{O}} \mapsto e^{-\sigma \Delta} \hat{\mathcal{O}}
$$

Because of the gauge invariance Eq. (2.4) the fields $\tau$ and $g_{\mu \nu}$ are not uniquely defined, but we will simply choose canonical background field configurations $g_{\mu \nu}$ and $\tau$. For our main applications, $g_{\mu \nu}=\eta_{\mu \nu}$ so that we are considering a conformally flat background. Correlation functions of $\tau$ then define dilaton correlation functions in flat spacetime, which will be the main object of study in the following.

The UV theory is invariant under Weyl transformations up to the Weyl anomaly [10], so we have

$$
\begin{aligned}
W\left[\hat{g}_{\mu \nu}\right]=W_{\mathrm{UV}} & {\left[g_{\mu \nu}\right]-\int d^{4} x \sqrt{-g} \tau\left[-a_{\mathrm{UV}} E_{4}(g)+c_{\mathrm{UV}} W^{2}(g)\right]+O\left(\tau^{2}\right) } \\
& + \text { relevant terms. }
\end{aligned}
$$

The terms higher order in $\tau$ complete themselves into the WZ term:

$$
W\left[\hat{g}_{\mu \nu}\right]=W_{\mathrm{UV}}\left[g_{\mu \nu}\right]-S_{\mathrm{WZ}}\left[g_{\mu \nu}, \tau ; a_{\mathrm{UV}}, c_{\mathrm{UV}}\right]+\text { relevant terms. }
$$

\footnotetext{
${ }^{1}$ We follow the conventions of KS for the metric and dilaton.
} 
The WZ term is given $b^{2}$

$$
\begin{aligned}
S_{\mathrm{WZ}}\left[g_{\mu \nu}, \tau ; a, c\right]=\int d^{4} x \sqrt{-g}\{- & a\left[\tau E_{4}(g)\right. \\
& +4\left(R^{\mu \nu}(g)-\frac{1}{2} g^{\mu \nu} R(g)\right) \Omega^{-2} \partial_{\mu} \Omega \partial_{\nu} \Omega \\
& \left.+4 \Omega^{-3}(\partial \Omega)^{2} \square \Omega-2 \Omega^{-4}(\partial \Omega)^{4}\right] \\
& \left.+c \tau W^{2}(g)\right\}
\end{aligned}
$$

where

$$
\Omega=e^{-\tau}
$$

The reason that the full WZ term appears in Eq. (2.7) is that it is the unique term that correctly reproduces the abelian nature of conformal transformations. In the present context, this means that the right-hand side of Eq. (2.7) is invariant under the gauge transformations Eq. (2.4):

$$
\begin{aligned}
\delta S_{\mathrm{WZ}}\left[g_{\mu \nu}, \tau ; a_{\mathrm{UV}}, c_{\mathrm{UV}}\right] & =\int d^{4} x \sqrt{-g} \alpha\left[-a_{\mathrm{UV}} E_{4}(g)+c_{\mathrm{UV}} W^{2}(g)\right] \\
& =\delta W\left[g_{\mu \nu}\right] .
\end{aligned}
$$

Eq. (2.7) is basis for all the results in this paper. It shows that the dependence on $\tau$ in the UV comes entirely through the WZ term. Note that even in flat space, the WZ term contains dilaton self-interaction terms, which will be crucial for the argument. The relevant deformation terms depend on $\tau$, but these are unimportant in the UV.

Note that if the IR theory is given by a CFT with only irrelevant deformations, exactly the same logic also gives

$$
W\left[\hat{g}_{\mu \nu}\right]=W_{\mathrm{IR}}\left[g_{\mu \nu}\right]-S_{\mathrm{WZ}}\left[g_{\mu \nu}, \tau ; a_{\mathrm{IR}}, c_{\mathrm{IR}}\right]+\text { irrelevant terms, }
$$

\footnotetext{
${ }^{2}$ We will be interested in the WZ term in flat spacetime as a function of the dilaton field. It is therefore worth noting that in flat spacetime the WZ term is the unique term in the dilaton Lagrangian that is invariant under special conformal transformations only up to a total derivative term. (A classification of dilaton invariants was given in Ref. [11].) The other terms in the dilaton effective action can be written in terms of $\hat{g}_{\mu \nu}$ and are therefore exactly invariant. This is analogous to the anomaly in the chiral Lagrangian for a $G / H$ coset, where the WZ term is the unique term that shifts by a total derivative under $G$ transformations.
} 
where $W_{\mathrm{IR}}$ is the effective action for the IR CFT with anomaly coefficients $a_{\mathrm{IR}}$ and $c_{\mathrm{IR}}$. We will show below that in general the IR CFT contains relevant deformation terms that give a nonlocal relevant correction to the right-hand side of Eq. (2.11). However, we will show that $a_{\mathrm{IR}}$ is nonetheless directly related to the on-shell dilaton scattering amplitude, the crucial observable in the KS argument.

We now return to the UV theory and consider the dilaton couplings arising from the relevant deformation terms. The original action is written in terms of CFT fields $\hat{\Phi}$ and background metric $\hat{g}_{\mu \nu}$. Defining

$$
\hat{g}_{\mu \nu}=g_{\mu \nu} e^{-2 \tau}, \quad \hat{\Phi}=e^{\tau \Delta_{\Phi}} \Phi
$$

the soft terms in the action have the form

$$
S_{\text {soft }}\left[\hat{\Phi}, \hat{g}_{\mu \nu}\right]=\int d^{4} x \sqrt{-g}(m \Omega)^{4-\Delta} \mathcal{O}\left[\Phi, g_{\mu \nu}\right]
$$

We see that if we change variables to make decoupling manifest, we automatically nonlinearly realize the Weyl invariance in terms of $g_{\mu \nu}$ and $\tau$. This gives a new perspective on the nonlinear realization of conformal symmetry used in the KS argument.

\subsection{Dilaton Effective Field Theory}

In this section we are assuming that the IR dynamics is controlled by a different CFT, so we can apply the same steps to the IR theory. The IR theory is to be regarded as an effective field theory, and we must include all couplings allowed by symmetries. We are particularly concerned about possible relevant terms, since these dominate in the IR. ${ }^{3}$

The observable we will be interested in is dilaton-dilaton scattering in flat spacetime, in a low-energy expansion. Specifically, we define the physical dilaton field $\varphi$ by

$$
\Omega=1+\frac{\varphi}{f}
$$

where $f$ is the dilaton decay constant. The WZ term Eq. (2.8) contains $O\left(E^{4}\right)$ dilaton self-couplings. We want to see if there are other terms in the effective theory that

\footnotetext{
${ }^{3}$ For the UV theory we would also like to know the most general relevant deformations allowed by symmetries in order to study the most general theory that flows to the UV CFT in the UV. The analysis below is relevant to this case as well.
} 
are more important in the IR. We will show that the interactions in the WZ term dominate at low energies provided that we impose the on-shell condition $\square \varphi=0$, or

$$
\square \Omega=0
$$

on the external dilaton lines, and fine-tune the IR cosmological constant. The cubic dilaton interaction in the WZ term then vanishes, but the quartic term remains. There are other possible definitions of the dilaton field and on-shell condition, but we will show that this choice is the one that ensures that the WZ term dominates the amplitude.

In the IR effective theory $m$ is a UV cutoff scale, and an operator $\mathcal{O}$ with dimension $\Delta$ in the IR CFT is to be viewed as order $E^{\Delta}$ in the low-energy expansion of dilaton interactions in flat spacetime. (CFT operators are defined to have definite dimensions, and have vanishing VEV in flat spacetime.) The most general relevant dilaton couplings to the CFT have the form

$$
S_{1}[\mathcal{O}]=\int d^{4} x \sqrt{-g}(m \Omega)^{4-\Delta} \mathcal{O}
$$

and

$$
\begin{aligned}
S_{2}[\mathcal{O}] & =\int d^{4} x \sqrt{-\hat{g}} m^{2-\Delta} R(\hat{g}) \hat{\mathcal{O}} \\
& =\int d^{4} x \sqrt{-g}(m \Omega)^{2-\Delta}\left(R(g)-6 \Omega^{-1} \square \Omega\right) \mathcal{O},
\end{aligned}
$$

where $\mathcal{O}$ is a scalar primary operator with dimension $\Delta$. In the low-energy expansion

$$
S_{1}=O\left(m^{4-\Delta} E^{\Delta}\right), \quad S_{2}=O\left(m^{2-\Delta} E^{2+\Delta}\right),
$$

so $S_{1}$ is relevant for $\Delta<4$, and $S_{2}$ is relevant for $\Delta<2$. It is easily seen that there are no other relevant couplings. If $\mathcal{O}$ is a non-primary operator then Eq. (2.16) is a total derivative and Eq. (2.17) is irrelevant, since unitarity bounds imply

$$
\Delta(\square \mathcal{O})>3, \quad \Delta\left(\nabla_{\mu} \mathcal{J}^{\mu}\right)>4, \quad \Delta\left(\nabla_{\mu} \nabla_{\nu} \mathcal{T}^{\mu \nu}\right)>6
$$

etc. We can write other dilaton couplings involving higher spin operators such as

$$
\Delta S=\int d^{4} x \sqrt{-\hat{g}}\left[m^{1-\Delta} \hat{\nabla}_{\mu} R(\hat{g}) \hat{\mathcal{J}}^{\mu}+m^{2-\Delta} R_{\mu \nu}(\hat{g}) \hat{\mathcal{T}}^{\mu \nu}\right]
$$

but these are also irrelevant by the unitarity bounds.

Note that the IR CFT always has at least one relevant operator, namely the identity operator $\mathcal{O}=1$. The term $S_{1}[\mathcal{O}=1]$ is a cosmological constant term that 
gives contributions to dilaton-dilaton scattering that are larger than the WZ term. We eliminate this by tuning the IR cosmological constant to zero. The term $S_{2}[\mathcal{O}=1]$ is a kinetic term for the dilaton. It is quadratic in $\Omega$ (and hence $\varphi$ ), and therefore does not contribute to dilaton scattering.

If the IR CFT contains relevant operators $\mathcal{O} \neq 1, S_{1}[\mathcal{O}]$ must be fine-tuned away, otherwise the CFT does not describe the asymptotic behavior of the theory in the IR as assumed. This leaves $S_{2}[\mathcal{O}]$, which gives a relevant coupling of the dilaton to the CFT for $\Delta<2$. This cannot be fine-tuned away in general, but it does not affect the dynamics of the CFT in flat spacetime, and it does not contribute to dilaton scattering if we impose the on-shell condition Eq. (2.15). Other possible effects of these terms will be discussed in $§ 2.3$ below.

Note that imposing the on-shell condition Eq. (2.15) is equivalent to solving the equations of motion that result from varying the kinetic term $S_{2}[\mathcal{O}=1]$. We could therefore define the amplitude more physically by giving a large coefficient $f^{2}$ to the kinetic term and expanding in inverse powers of $1 / f$. This gives a nice physical interpretation to the dilaton scattering amplitude, but is not strictly necessary for our argument. We prefer to emphasize that nowhere in our arguments does the dilaton need to be dynamical.

Finally, we must consider 4-derivative terms that depend only on the dilaton. At 4 derivatives, there are three independent Weyl invariant terms that may be written

$$
\Delta S=\int d^{4} x \sqrt{-\hat{g}}\left[E_{4}(\hat{g})+W^{2}(\hat{g})+R^{2}(\hat{g})\right] .
$$

These however do not contribute to dilaton scattering in flat spacetime: the first term is a total derivative; the second vanishes identically on conformally flat metrics; and the third has the form

$$
\sqrt{-\hat{g}} R^{2}(\hat{g})=\sqrt{-g}\left[R(g)-6 \Omega^{-1} \square \Omega\right]^{2},
$$

which does not contribute if we impose the on-shell condition Eq. (2.15).

We have therefore shown that the leading contribution to the dilaton scattering amplitude comes from the WZ term Eq. (2.8).

\subsection{Operators with $\Delta \leq 2$}

We have seen above that if there is a scalar primary operator $\mathcal{O}$ with dimension $\Delta<2$ $(\Delta=2)$, the theory has a relevant (marginal) interaction term of the form

$$
\Delta S=\int d^{4} x \sqrt{-\hat{g}} m^{2-\Delta} R(\hat{g}) \hat{\mathcal{O}}
$$


These terms do not affect the dilaton-dilaton scattering amplitude in flat spacetime on-shell, because $R(\hat{g})=0$ for these backgrounds. On the other hand, they do give IR singular contributions to the correlation functions of $T^{\mu \nu}$. In this section we will discuss in more detail these operators and make it more clear that they do not invalidate our arguments.

If $\hat{\mathcal{O}}$ is a singlet under all symmetries, then the theory is highly unnatural as an IR CFT. However, there is one important special case where a theory with such an operator appears in the IR, namely theories with Nambu-Goldstone bosons. These are free massless scalars $\pi$ in the IR, and the absence of a mass for the scalars is rendered natural by a $\pi$ shift symmetry. This shift symmetry also forbids an improvement term of the form $\pi^{2} R(g)$ that makes the stress-energy tensor conformal. We can write this theory as a conformal scalar plus an "unimprovement" term $-\frac{1}{12} \pi^{2} R(\hat{g})$, where $\pi^{2}$ is a primary operator of dimension $\Delta=2$.

To understand the effects of the term Eq. (2.23) on the off-shell dilaton amplitudes, note that it contains at least one power of the dilaton field $\varphi$. This means that the $n$-dilaton amplitude involves at most $n$ insertions of the term Eq. (2.23). If $\Delta<2$, the term with the most insertions of the interaction is the most relevant, so e.g. the dilaton-dilaton scattering amplitude is

$$
A\left(p_{1}, \ldots, p_{4}\right) \sim\left(\frac{m^{2-\Delta}}{f}\right)^{4} p_{1}^{2} \cdots p_{4}^{2}\left\langle\mathcal{O}\left(p_{1}\right) \cdots \mathcal{O}\left(p_{4}\right)\right\rangle
$$

The correlation function of $\mathcal{O}$ is to be evaluated in the unperturbed CFT. We see that the amplitude is singular in the IR, but vanishes on-shell, as it must. We can extend this logic to correlation functions of the full stress-energy tensor $T^{\mu \nu}$. The term Eq. (2.23) vanishes in flat spacetime, so the $n$-point functions of $T^{\mu \nu}$ in the perturbed theory are given by a finite sum of $2, \ldots, n$ point functions of the unperturbed theory. This clarifies the relationship between the anomaly in the perturbed and the unperturbed theory, namely the anomalous terms in the perturbed theory are determined by the anomaly of the unperturbed theory in a straightforward way.

It should be stressed that the extra terms discussed above correspond to genuinely nonlocal effects in the quantum effective action $W\left[\hat{g}_{\mu \nu}\right]$. This is not surprising since Eq. (2.23) is an explict IR breaking of conformal invariance. We can see the nonlocal structure by direct calculation of the quantum effective action in some simple cases. For example, in the simplest case where $\hat{\mathcal{O}}=\Phi$ is a free scalar field with $\Delta=1$ the generated term is

$$
\Delta W \sim \int d^{4} x \sqrt{-\hat{g}} m^{2} R(\hat{g}) \frac{1}{\hat{\square}} R(\hat{g})
$$


while in the case $\hat{\mathcal{O}}=\pi^{2}$ where $\pi$ is a free massless Goldstone boson we get a series of terms

$$
\Delta W \sim \int d^{4} x \sqrt{-\hat{g}} R(\hat{g})(\ln \hat{\square}) R(\hat{g})+\cdots
$$

The Weyl variation of these term is also non-local. That is not a contradiction since Eq. (2.23) explicitly breaks Weyl invariance. However an on-shell dilaton is beautifully insenstive to this effect.

We also remark that in the presence of the term Eq. (2.23), the stress-energy tensor defined by variation with respect to the metric is not the canonical energy momentum tensor of the IR CFT (which is a primary spin 2 field). It differs from it by mixing with descendants of $\hat{\mathcal{O}}$. Roughly one has

$$
T^{\mu \nu}=T_{\mathrm{IRCFT}}^{\mu \nu}+m^{2-\Delta}\left(\partial^{\mu} \partial^{\nu}-\square \eta^{\mu \nu}\right) \mathcal{O}+\cdots
$$

However, all of these effects do not matter in backgrounds with $\square \Omega=0$.

Finally, we show that even if operators with $\Delta \leq 2$ can be somehow tuned away in the action, they give rise to IR divergences in the off-shell dilaton-dilaton scattering amplitude. We have

$$
\begin{aligned}
A\left(p_{1}, \ldots, p_{4}\right) & =\frac{\delta^{4} W}{\delta \varphi\left(p_{1}\right) \cdots \delta \varphi\left(p_{4}\right)} \\
& =\left\langle T\left(p_{1}\right) T\left(p_{2}\right) T\left(p_{3}\right) T\left(p_{4}\right)\right\rangle+\text { contact terms. }
\end{aligned}
$$

For the forward amplitude $p_{1}=-p_{3}, p_{2}=-p_{4}$, the potential divergence is in the zero momentum channel, corresponding to $x_{1}-x_{3} \sim x_{2}-x_{4} \ll x_{3}-x_{4}$. Let us analyze this first in the regime $x_{1}-x_{3} \sim x_{2}-x_{4} \gg m^{-1}$. In the effective IR CFT the trace has dimension $\Delta_{\mathrm{IR}}>4$, so the OPE for the two close pairs gives

$$
\begin{aligned}
A \propto m^{16-4 \Delta_{\mathrm{IR}}} \int & d^{4} x_{1} d^{4} x_{2} d^{4} x_{3} e^{i\left[p_{1} \cdot\left(x_{1}-x_{3}\right)+p_{2} \cdot\left(x_{2}-x_{4}\right)\right]} \\
& \times\left(x_{1}-x_{3}\right)^{\Delta-2 \Delta_{\mathrm{IR}}}\left(x_{2}-x_{4}\right)^{\Delta-2 \Delta_{\mathrm{IR}}}\left\langle\mathcal{O}\left(x_{3}\right) \mathcal{O}\left(x_{4}\right)\right\rangle \\
\propto m^{16-4 \Delta_{\mathrm{IR}}}( & \left.m^{2 \Delta_{\mathrm{IR}}-\Delta-4}-p_{1}^{2 \Delta_{\mathrm{IR}}-\Delta-4}\right)\left(m^{2 \Delta_{\mathrm{IR}}-\Delta-4}-p_{2}^{2 \Delta_{\mathrm{IR}}-\Delta-4}\right) \\
& \times \int d^{4} x_{3}\left(x_{3}-x_{4}\right)^{-2 \Delta_{\mathcal{O}}},
\end{aligned}
$$

where $\mathcal{O}$ is the operator of lowest dimension $\Delta$ in $T T$. In each parenthesis, the term $m^{2 \Delta_{\mathrm{IR}}-\Delta-4}$ comes from the UV end of the integral. This term cannot actually be present it corresponds to a effective interaction proportional to $m^{4-\Delta} \mathcal{O}$ in the IR CFT coupled to a background metric. Since the IR is a CFT this must be absent, canceled 
by the tuning that makes the IR theory conformal. The remaining contribution is proportional to positive powers of $p_{1}^{2}$ and $p_{2}^{2}$ : we see that if $\Delta \leq 2$ the off-shell amplitude diverges, but the on-shell amplitude is IR finite. (The apparent divergence at $p_{i}^{2}=0$ for $\Delta>4$ is not real: it comes from $x_{1}-x_{3}, x_{2}-x_{4} \gg x_{3}-x_{4}$ where the OPE is not valid.) The regime $x_{1}-x_{3} \sim x_{2}-x_{4} \lesssim m^{-1}$ may similarly bring in powers of the UV cutoff, which must be absent by renormalization.

\subsection{Dispersive Argument}

We now consider dilaton scattering at low energy. Specifically, we are interested in the amplitude for $\varphi \varphi \rightarrow \varphi \varphi$ as a function of the Mandelstam variable $s$, with $t \rightarrow 0$. We denote this by $A(s)$. To define the amplitude, it is convenient to use our freedom to add UV counterterms to the theory that depend only on $\hat{g}_{\mu \nu}$. We will cancel the cosmological constant in the IR by adding a suitable counterterm, and we will add a WZ term to cancel the WZ term induced from the UV theory. That is, we compute the dilaton scattering amplitude using

$$
W\left[\hat{g}_{\mu \nu}\right]+S_{\mathrm{WZ}}\left[g_{\mu \nu}, \tau ; a_{\mathrm{UV}}, c_{\mathrm{UV}}\right]
$$

This gives an amplitude with good behavior in the UV and IR, allowing the use of unsubtracted dispersion relations to establish the positivity of the amplitude. Alternatively, we could use a subtracted dispersion relation on the amplitude defined by $W\left[\hat{g}_{\mu \nu}\right]$.

The leading behavior as $s \rightarrow 0$ of the amplitude is then

$$
A(s) \rightarrow \alpha \frac{s^{2}}{f^{4}}+O\left(\frac{m^{2\left(4-\Delta_{\mathrm{IR}}\right)} s^{\Delta_{\mathrm{IR}}-2}}{f^{4}}\right),
$$

where second term arises from the coupling of the dilaton to the IR CFT. Here $\Delta_{\text {IR }}>4$ is the lowest dimension of the irrelevant operators appearing in the deformation of the IR CFT. Note that if we had not canceled the cosmological constant term in the IR, the leading behavior of the amplitude would be $\sim s^{0}$.

As $s \rightarrow \infty$ the UV behavior is dominated either by the largest dimension relevant deformation with dimension $\Delta_{\mathrm{UV}}$, or by the cosmological constant term:

$$
A(s) \sim \begin{cases}\frac{m^{2\left(4-\Delta_{\mathrm{UV}}\right)} s^{\Delta_{\mathrm{UV}}-2}}{f^{4}} & \text { if } \Delta_{\mathrm{UV}} \geq 2, \\ \frac{m^{4}}{f^{4}} & \text { if } \Delta_{\mathrm{UV}}<2 .\end{cases}
$$

Note that if we had not subtracted the UV WZ term, the leading behavior of the amplitude would be $\sim s^{2}$. 


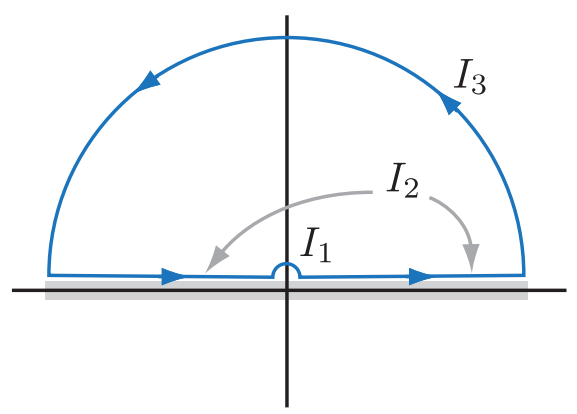

Fig. 2. The integration contour in the complex $s$ plane used to argue that $\alpha>0$.

With this definition of the amplitude, the coefficient of the WZ term in the IR is $a_{\mathrm{UV}}-a_{\mathrm{IR}}$, so we have

$$
\alpha=8\left(a_{\mathrm{UV}}-a_{\mathrm{IR}}\right)
$$

Therefore, the $a$-theorem is equivalent to $\alpha>0$.

Theories with $\alpha<0$ have superluminal propagation of dilaton excitations in certain nontrivial background dilaton configurations, and are therefore acausal. However, in order for this to be a physical problem, the dilaton must be a propagating degree of freedom. The acausality of this theory may simply be a sign that we cannot UV complete the theory with a dynamical dilaton. ${ }^{4}$ In fact, we do not know of any non-supersymmetric UV completion of a theory with a dynamical dilaton.

We will give a rigorous dispersive argument that $\alpha>0$ without assuming anything about the UV completion of the theory. We consider the contour integral

$$
0=\frac{1}{2 \pi i} \oint d s \frac{A(s)}{s^{3}}
$$

along the contour shown in Fig. 2. The integral $I_{1}$ over a small semi-circle of radius $\epsilon$ is given by

$$
I_{1}=-\frac{\alpha}{2 f^{4}}+O\left(\epsilon^{\Delta_{\mathrm{IR}}-4}\right) .
$$

Because $\Delta_{\mathrm{IR}}>4$, the $\epsilon \rightarrow 0$ limit picks out the coefficient of the leading low-energy behavior of the scattering amplitude.

\footnotetext{
${ }^{4}$ In fact, note that we can get any value for $\alpha$ by choosing the WZ counterterm in the UV arbitrarily. The choices that give $\alpha<0$ presumably do not have causal UV completions.
} 
We now turn to $I_{2}$. The function $A(s)$ has cuts all along the real $s$ axis due to massless CFT intermediate states. By crossing symmetry $A(s)=A(u)=A(-s)$, and so the contribution from the integral along the real axis is

$$
I_{2}=\frac{1}{\pi} \int_{\epsilon}^{\infty} d s \frac{\operatorname{Im} A(s)}{s^{3}}=\frac{1}{\pi} \int_{\epsilon}^{\infty} d s \frac{\sigma(s)}{s^{2}},
$$

where $\sigma$ is the total cross section for $\varphi \varphi \rightarrow$ CFT in the probe limit. The integral $I_{2}$ is manifestly positive, so we have proved $\alpha>0$ (and hence the $a$-theorem) provided that we can neglect the contribution from the large semicircle. In fact, the UV behavior Eq. (2.32) is precisely sufficient to ensure that this is the case.

This completes the proof of the $a$-theorem in the case where the UV CFT is deformed by relevant operators, and the IR CFT by irrelevant operators. In the remainder of this section, we consider the important special case where the flow in the UV or IR is logarithmic, induced by marginally relevant or irrelevant operators. For example, we may be interested in an asympotically free gauge theory in the UV, or theories with $U(1)$ gauge factors in the IR. We will show very generally that the dilaton decouples sufficiently rapidly that we can apply the dispersive argument above to these cases. The issue amounts to the UV and IR convergence of the integral $I_{2}$, since the IR convergence implies that $I_{1}$ is given by Eq. (2.35), and the UV convergence implies $I_{3}=0$.

We consider first the case where the UV theory is an asymptotically free gauge theory. This theory requires a UV cutoff $\Lambda$, which breaks scale (and conformal) invariance and induces a coupling to the dilaton. The dependence on $\Lambda$ is governed by the RG equation

$$
\frac{d}{d \ln \Lambda}\left(\frac{1}{g^{2}}\right)=b+O\left(g^{2}\right)
$$

with $b>0$. We write the UV Lagrangian as

$$
\mathcal{L}_{\mathrm{UV}}=-\frac{1}{4 g^{2}} F_{\mu \nu}^{2}+\text { regulator terms. }
$$

where $F_{\mu \nu}$ has no $g$ dependence. We can couple the dilaton to make the theory scale invariant by making the replacement

$$
\Lambda \rightarrow \Lambda \Omega
$$

in the regulator terms. The dependence on the dilaton then follows from the the RG:

$$
\begin{aligned}
\mathcal{L}_{\mathrm{UV}} & \rightarrow \mathcal{L}_{\mathrm{UV}}+\left[\frac{d}{d \ln \Lambda}\left(\frac{1}{g^{2}}\right) \ln \Omega+\frac{1}{2} \frac{d}{d(\ln \Lambda)^{2}}\left(\frac{1}{g^{2}}\right) \ln ^{2} \Omega+\cdots\right] F_{\mu \nu}^{2} \\
& =\mathcal{L}_{\mathrm{UV}}+\left[-\frac{b}{4} \ln \Omega+O\left(g^{2}\right)\right] F_{\mu \nu}^{2} .
\end{aligned}
$$


When expanded in $\varphi$, this coupling contains all powers of the dilaton excitations. The quantity of interest is the cross section for two dilatons to scatter into two gauge bosons. We canonically normalize the gauge fields by $A_{\mu} \rightarrow g A_{\mu}$ and find the amplitude $\mathcal{M}(\varphi \varphi \rightarrow A A) \sim b g^{2} / f^{2}$. For $s \rightarrow \infty$ we therefore have

$$
\sigma(\varphi \varphi \rightarrow A A) \sim \frac{b^{2} g^{4}(s)}{f^{4}} s
$$

The solution of the RG equation Eq. (2.37) is $g^{2}(s) \sim 1 / \ln s$, so the integral $I_{2}$ has the large $s$ behavior

$$
I_{2} \sim \int^{\infty} \frac{d \ln s}{(\ln s)^{2}}
$$

which converges in the UV. For $I_{3}$ we need the large- $s$ behavior of the real as well as the imaginary part of $A$. In order to get the correct imaginary part we must have $\operatorname{Re}(A) \sim \frac{s^{2}}{\ln s}$, which is just sufficient to give $I_{3}=0$.

A similar argument holds if the IR theory has a gauge coupling that runs to zero in the IR. In that case, $g^{2}(s) \sim 1 / \ln s^{-1}$ as $s \rightarrow 0$, and we have

$$
\operatorname{Im} A(s)-\frac{\alpha s^{2}}{f^{4}} \sim \frac{b^{2} g^{4}(s)}{f^{4}} s^{2} \sim \frac{s^{2}}{\left(\ln s^{-1}\right)^{2}} .
$$

The integral therefore has the small $s$ behavior

$$
I_{2} \sim \int_{0} \frac{d \ln s^{-1}}{\left(\ln s^{-1}\right)^{2}},
$$

which is sufficient for convergence in the IR. If the one-loop beta function vanishes, we have

$$
\frac{d}{d \ln \Lambda}\left(\frac{1}{g^{2}}\right)=b^{\prime} g^{2}
$$

with $b^{\prime}>0$. In this case, the amplitude $\mathcal{M}(\varphi \varphi \rightarrow A A) \sim b^{\prime} g^{4} / f^{2}$ and we have as $s \rightarrow 0$

$$
A(s)-\frac{\alpha s^{2}}{f^{4}} \sim \frac{b^{\prime 2} g^{8}(s)}{f^{4}} s^{2} \sim \frac{s^{2}}{\left(\ln s^{-1}\right)^{2}},
$$

as before.

Gauge theory is an important special case, but it is somewhat unusual from the CFT point of view because the dimension 4 operator that generates the flow is $F_{\mu \nu}^{2}$, and the free limit corresponds to an infinite coefficient for this operator in the action. 
We therefore consider the effect of adding a dimension 4 operator $\mathcal{O}$ with coefficient $\lambda$ to either the UV or the IR CFT:

$$
\Delta \mathcal{L}=\lambda \mathcal{O}
$$

For small $\lambda$ the RG equation has the form

$$
\frac{d \lambda}{d \ln \Lambda}=b \lambda^{n}
$$

For example for a $\Phi^{4}$ term $n=2$, while for a Yukawa coupling $n=3$. The dilaton coupling follows from the replacement $\Lambda \rightarrow \Lambda(1-\varphi / f)$, so we have $A(\varphi \varphi \rightarrow$ QFT $) \sim$ $b \lambda^{n} / f^{2}$ and hence

$$
\sigma(\varphi \varphi \rightarrow \mathrm{QFT}) \sim \frac{b^{2} \lambda^{2 n}(s)}{f^{4}} s .
$$

For the case where $b>0$ and this operator appears in the IR CFT, we have as $s \rightarrow 0$

$$
\lambda(s) \sim\left(\frac{1}{\ln s^{-1}}\right)^{1 /(n-1)}
$$

and the integral $I_{2}$ has the form as $s \rightarrow 0$

$$
I_{2} \sim \int \frac{d \ln s^{-1}}{\left(\ln s^{-1}\right)^{2 n /(n-1)}} .
$$

This always converges as $s \rightarrow 0$ because $2 n /(n-1)>2$ for $n>1$. In the case where the operator $\mathcal{O}$ appears in the UV CFT, a similar argument proves the UV convergence. Similarly one can see that $\left|A(s) / s^{2}\right| \rightarrow 0$ at infinity, ensuring $I_{3}=0$. We conclude that the convergence of the dispersion relation holds very generally even with logarithmic flows in the UV or IR.

\subsection{Gravitational Anomalies}

We have assumed that it is possible to couple to a background metric in a diffeomorphism invariant way. This is not possible if there is $U(1)$ gauge symmetry with $\operatorname{Tr} Q \neq 0$, where $Q$ is the gauge charge. In such theories, the gauge current is not conserved if we require diffeomorphism invariance:

$$
\partial_{\mu} J^{\mu}=-\frac{\operatorname{tr}(Q)}{384 \pi^{2}} R \tilde{R}
$$


where $R \tilde{R}=\frac{1}{2} \epsilon^{\mu \nu \rho \sigma} R_{\mu \nu}{ }^{\tau \omega} R_{\rho \sigma \tau \omega}$. Alternatively, we can maintain gauge invariance at the price of diffeomorphism invariance by using the fact that

$$
R \tilde{R}=\partial_{\mu} K^{\mu}
$$

where $K^{\mu}$ is not generally covariant. We add to the diffeomorphism invariant action a term

$$
\Delta S=\frac{\operatorname{tr}(Q)}{384 \pi^{2}} \int d^{4} x \sqrt{-g} A_{\mu} K^{\mu} .
$$

The fact that this term violates diffeomorphism invariance is not a fundamental problem for us, since the metric is just a background field. It does mean that we must take care in defining the background metric, since it no longer has a geometrical meaning. For our purposes, it is sufficient to write

$$
g_{\mu \nu}=e^{-2 \tau} \eta_{\mu \nu}
$$

where $\eta_{\mu \nu}$ is the flat metric in Cartesian coordinates and $\tau$ is the dilaton field.

Now the point is that the additional term Eq. (2.54) does not contain any coupling of the dilaton. The reason is that for the background metric $e^{-2 \tau} \eta_{\mu \nu}$ this coupling must respect global Lorentz invariance. It is a polynomial involving $\epsilon^{\mu \nu \rho \sigma}, A_{\mu}, 3$ derivatives, and powers of $\varphi$. It is easy to see that there is no such Lorentz invariant, and so the dilaton coupling vanishes identically. The presence of this term therefore does not affect the arguments of this paper, very much like the improvement terms for scalars, and the $R^{2}$ counterterms.

Alternatively, we can avoid breaking diffeomorphism invariance by introducing a background 2-form field field $B_{\mu \nu}$, and restore diffeomorphism invariance by the Green-Schwarz mechanism [12]. The anomalous variation is of the form

$$
\delta W \propto \int F \wedge \operatorname{Tr}(\delta \lambda R)
$$

where $\delta \lambda$ is a local Lorentz transformation. Introduce an interaction $\int F \wedge B$. This is invariant under the usual 2-form transformation $\delta B=d \xi$, and with the transformation $\delta B=-\operatorname{Tr}(\delta \lambda R)$ it cancels the anomaly. The complication is that one must now consider possible gauge-invariant interactions built out of the field strength $H=d B+\omega_{3 \mathrm{~L}}$, where $\omega_{3 \mathrm{~L}}$ is the gravitational Chern-Simons term. In fact, $H$ vanishes in the background $B=0, \hat{g}=\eta e^{-2 \tau}$, and so the extra terms do not contribute to dilaton amplitudes. 


\section{Restrictions on Perturbative RG Flows}

We now generalize these arguments to restrict perturbative RG flows. We will show that in unitary theories the only IR or UV asymptotics that can be described in pertrubation theory is conformal invariance. Our arguments in fact apply to small perturbations of a conformal fixed point, even if it strongly coupled. Closely related monotonicity results for perturbative flows were obtained in Refs. [7, 8, 9].

\subsection{General Framework}

Our argument is based on the same amplitude $A(s)$ defined above. We work with the dimensionless function $\alpha(s)$ defined by

$$
A(s)=\frac{\alpha(s) s^{2}}{f^{4}} .
$$

It will be essential to our argument below that this amplitude has no counterterm, and is therefore calculable purely in terms of the renormalized couplings in the theory; this follows from the analysis in $\S 2$ of possible dilaton couplings. To reiterate the basic idea, we have included the most general diffeomorphism invariant counterterms depending on the background metric $\hat{g}_{\mu \nu}$, and these do not affect the dilaton amplitude. The split into $g_{\mu \nu}$ and $\tau$ used to define the dilaton amplitude is arbitrary; the theory only depends on $\hat{g}_{\mu \nu}$. The WZ term parameterizes the effects of the anomaly and does not represent a counterterm.

In free field theory the function $\alpha(s)$ equals -8 times the (constant) coefficient of the WZ term. The discussion of $\S 2.2$ showed that there is no counterterm for the amplitude $A(s)$, and therefore all divergences in $\alpha(s)$ can be absorbed into renormalization of the renormalized couplings of the theory. When interactions are turned on, $\alpha(s)$ will thus be written as a power series in the renormalized couplings evaluated at a renormalization scale $\mu \sim s^{1 / 2}$. The same structure holds for marginal perturbations around any conformal fixed point, with the couplings replaced by the coefficients of the marginal operators in the perturbation.

In this section we are considering flows that remain perturbative in the UV or IR limit, that is, the coupling remains in some bounded neighborhood of the origin. The magnitude of $\alpha(s)$ is then bounded. This is the key observation that allows us to obtain new constraints on RG flows.

On the closed contour $C$ of Fig. 2 we have

$$
\frac{1}{i \pi} \int_{C} \frac{d s}{s} \alpha(s)=0
$$


Let the radii of the inner and outer semicircles be $\sigma_{1}$ and $\sigma_{3}$, respectively. Then

$$
\bar{\alpha}\left(\sigma_{3}\right)-\bar{\alpha}\left(\sigma_{1}\right)=-\frac{2}{\pi} \int_{\sigma_{1}}^{\sigma_{3}} \frac{d s}{s} \operatorname{Im} \alpha(s),
$$

where $\bar{\alpha}(\sigma)$ is the average value of $\alpha(s)$ on a semi-circular contour of radius $\sigma$. We have used $\alpha(-s)=\alpha(s)$, which implies $\operatorname{Im} \alpha\left(-s^{\prime}\right)=-\operatorname{Im} \alpha\left(s^{\prime}\right)$. We can also write this in a differential version

$$
\sigma \partial_{\sigma} \bar{\alpha}(\sigma)=-\frac{2}{\pi} \operatorname{Im} \alpha(\sigma)
$$

Now consider Eq. (3.3) as $\sigma_{1} \rightarrow 0$ with $\sigma_{3}$ fixed. The quantity $\bar{\alpha}\left(\sigma_{1}\right)$ is calculable in perturbation theory, and must therefore remain bounded as long as perturbation theory is valid. Therefore, the integral

$$
\alpha(s)=\frac{2}{\pi} \int_{\sigma_{1}}^{\sigma_{3}} d s^{\prime} \frac{\operatorname{Im} \alpha\left(s^{\prime}\right)}{s^{\prime}}+\text { finite }
$$

must remain bounded as $\sigma_{1} \rightarrow 0$. Similarly, by taking $\sigma_{3} \rightarrow \infty$ at fixed $\sigma_{1}$, we can conclude that the integral remains bounded as $\sigma_{3} \rightarrow \infty$. The boundedness of these integrals has strong implications in unitary theories, because for these $\operatorname{Im} \alpha(s)$ is a sum of positive terms, each of which is a squared amplitude connecting the vacuum with some $n$-particle state. In particular it follows immediately that $\operatorname{Im} \alpha(s)$ vanishes in both the UV and IR limits. ${ }^{5}$

In detail, we write the (in general off-shell) dilaton-dilaton scattering amplitude

$$
(2 \pi)^{4} \delta^{4}\left(p_{1}+\cdots+p_{4}\right) A\left(p_{1}, \ldots, p_{4}\right)=\frac{\delta^{4} W}{\delta \varphi\left(p_{1}\right) \cdots \delta \varphi\left(p_{4}\right)},
$$

where

$$
\begin{aligned}
f^{4} A\left(p_{1}, \ldots, p_{4}\right)=\langle & \\
& \left.\left(p_{1}\right) T\left(p_{2}\right) T\left(p_{3}\right) T\left(p_{4}\right)\right\rangle \\
& +\left\langle T\left(p_{1}+p_{2}\right) T\left(p_{3}\right) T\left(p_{4}\right)\right\rangle+\text { permutations } \\
& +\left\langle T\left(p_{1}+p_{2}\right) T\left(p_{3}+p_{4}\right)\right\rangle+\text { permutations } \\
& +\left\langle T\left(p_{1}+p_{2}+p_{3}\right) T\left(p_{4}\right)\right\rangle+\text { permutations } .
\end{aligned}
$$

(The average here means the time-ordered vacuum expectation value). We have defined the trace of the stress-energy tensor by differentiating the quantum effective action with respect to $\tau$ :

$$
\left\langle T\left(x_{1}\right) \cdots T\left(x_{n}\right)\right\rangle=\frac{1}{\sqrt{-g\left(x_{1}\right)}} \cdots \frac{1}{\sqrt{-g\left(x_{n}\right)}} \frac{\delta^{n} W\left[e^{-2 \tau} g_{\mu \nu}\right]}{\delta \tau\left(x_{1}\right) \cdots \delta \tau\left(x_{n}\right)} .
$$

\footnotetext{
${ }^{5}$ In a non-unitary theory, there would be the possibility that that the integral Eq. (3.5) converges due to rapid oscillations even if $\operatorname{Im} \alpha(s)$ is nonzero in the IR or UV.
} 
This definition coincides with the standard one,

$$
\left\langle T\left(x_{1}\right) \cdots T\left(x_{n}\right)\right\rangle=\frac{g^{\mu_{1} \nu_{1}}\left(x_{1}\right)}{\sqrt{-g\left(x_{1}\right)}} \cdots \frac{g^{\mu_{n} \nu_{n}}\left(x_{n}\right)}{\sqrt{-g\left(x_{n}\right)}} \frac{\delta^{n} W\left[g_{\mu \nu}\right]}{\delta g^{\mu_{1} \nu_{1}}\left(x_{1}\right) \cdots \delta g^{\mu_{n} \nu_{n}}\left(x_{n}\right)},
$$

up to contact terms. Several different powers of $T$ are present in the amplitude (3.7) because of the nonlinear relation (2.14) between $\varphi$ and $\tau$.

The imaginary part of $\alpha(s)$ is therefore

$$
\operatorname{Im} \alpha(s)=\sum_{X}\left|\left\langle X\left|T\left(p_{1}\right) T\left(p_{2}\right)+T\left(p_{1}+p_{2}\right)\right| 0\right\rangle\right|^{2},
$$

summed over all states of the CFT. We are considering theories of massless particles, but there are no IR divergences in Eq. (3.10) because these involve sums over all final states, i.e. it is completely inclusive.

To get constraints on the RG flow, we relate $\operatorname{Im} \alpha$ to the beta functions. For any perturbative theory, we have the operator identity

$$
T=\sum_{A} B_{A} \mathcal{O}^{A}+\mathcal{E}+\text { anomaly terms }
$$

The meaning of the various terms on the right-hand side is as follows. The operators $\mathcal{O}^{A}$ are a basis for the interaction terms in the Lagrangian

$$
\mathcal{L}_{\text {int }}=\sum_{A} \lambda_{A} \mathcal{O}^{A}
$$

and $B_{A}$ is a beta function associated with the coupling $\lambda_{A}$. We are following here the notation of Refs. $[7,8,9]$, which uses $B_{A}$ for the coefficients in $T$ when expressed in an operator basis where the divergence of currents, that in general appear on the right hand side of Eq. (3.11), are replaced by using the corresponding Ward identities. That determines a shift from the "naive" beta function $\beta_{A}$ to $B_{A}$ in the coefficients of $\mathcal{O}_{A}$. As already noticed in Ref. [9] ${ }^{6}$, and as explained in $\S 3.4$ below, $\beta_{A}$ have a degree of arbitrariness, which arises ultimately because the RG equation only involves $\int d^{4} x T$. This subtlety will not affect our argument, which only uses Eq. (3.11) and therefore only refers to $B_{A} \cdot{ }^{7}$

The term $\mathcal{E}$ in Eq. (3.11) represents terms that vanish by the equations of motion. These are important for contact terms, e.g.

$$
\langle\mathcal{E}(x) \mathcal{O}(y)\rangle \propto \delta^{4}(x-y)\langle\delta \mathcal{O}(x)\rangle
$$

\footnotetext{
${ }^{6}$ See for instance the discussion in the introduction and below Eq. (4.31) in that Ref..

${ }^{7}$ In version 1 of the present work, the notation $\beta_{A}$ was used for the coefficients in $T$. We have changed this to conform with the notation of Refs. $[7,8,9]$.
} 
where $\delta \mathcal{O}$ is the infinitesmal variation of $\mathcal{O}$ under scale transformations. The anomaly terms in Eq. (3.11) are proportional to

$$
a E_{4}(g)-c W^{2}(g)
$$

and are nonzero only for a nonzero gravitational field.

The operator identity Eq. (3.11) is in general valid for single insertions of $T$ in correlation functions involving arbitrarily many powers of elementary fields. When $T$ is inserted more than once, there are additional contact terms that can be thought of as additional insertions of scalar operators. In Eq. (3.10) these are important because of the appearance of the operator product $T\left(p_{1}\right) T\left(p_{2}\right)$. In $\S 3.2$ we avoid this complication by focusing on intermediate states $|X\rangle$ with nonzero angular momentum. In $\S 3.3$ to $\S 3.6$ we include also the effects of the $J=0$ states, giving somewhat sharper constraints.

We also note that the discussion in this section and the following ones can be directly generalized to perturbation theory around any conformal fixed point, whether free or strongly interacting. For perturbations around an IR fixed point, the $\Delta>4$ operators flow to zero and the $\Delta<4$ operators must be tuned away in order for the perturbative to describe the IR limit (like scalar mass terms in in the free conformal theory). The only nontrivial effects therefore come from $\Delta=4$ operators. $^{8}$ For perturbations around a UV fixed point, we must require that the $\Delta>4$ operators are not present, while the $\Delta<4$ operators flow to zero in the IR, so again the only nontrivial effects come from dimension-4 operators. For such theories, we expect Eq. (3.11) to hold, where $\mathcal{O}^{A}$ are a complete set of dimension-4 primary operators. Further details will be presented in Ref. [13].

\subsection{Constraints from Higher Partial Waves}

Restricting to $J>0$ partial waves eliminates both the contact interactions (3.13) and the linear $T$ term (3.10), leaving

$$
\begin{aligned}
\operatorname{Im} \alpha(s) & \geq \sum_{X, J_{X}>0}\left|\left\langle X\left|T\left(p_{1}\right) T\left(p_{2}\right)\right| 0\right\rangle\right|^{2} \\
& =\sum_{A, B}\left[B_{A}(s) B_{B}(s)\right]^{2} \sum_{X, J_{X}>0}\left|\left\langle X\left|\mathcal{O}^{A}\left(p_{1}\right) \mathcal{O}^{B}\left(p_{2}\right)\right| 0\right\rangle\right|^{2}
\end{aligned}
$$

\footnotetext{
${ }^{8}$ This can be generalized to operators where $\Delta-4$ is less than or of order the dimensionless quantity describing the perturbation, as is the case in Wilson-Fisher fixed points.
} 
This is a sum of positive terms, so each term must go to zero as $s \rightarrow 0$ and as $s \rightarrow \infty$. For example, we can consider only the $A=B$ terms and conclude that $B_{A} \rightarrow 0$ for all $A$ as $s \rightarrow 0$ provided that

$$
\left|\left\langle X\left|\mathcal{O}^{A}\left(p_{1}\right) \mathcal{O}^{A}\left(p_{2}\right)\right| 0\right\rangle\right|^{2} \neq 0
$$

as $s \rightarrow 0$ for some $X$ with $J \neq 0$. But this can easily seen to be the case by considering an intermediate state for which there is a tree-level matrix element with $\mathcal{O}^{A} \mathcal{O}^{A}$. For example, if $\mathcal{O}^{A}$ is a $\Phi^{4}$ interaction, we consider a tree level graph where the two operators are connected by a single propagator and the other legs connect to a six-scalar intermediate state: this contributes to all partial waves. Similarly, for $\mathcal{O}^{A}$ a Yukawa interaction a graph with a scalar exchange contribute for fourfermion intermediate states. Recalling that $\alpha(s)$ and the beta functions $B_{A}$ are all dimensionless, the sum over $X$ (implicitly including phase space integrals) will give a nonzero dimensionless constant for such states.

We conclude that $B_{A}$ must vanish for all $A$ as $s \rightarrow 0, \infty$, sufficiently rapidly that

$$
\int \frac{d s}{s} B_{A}^{2} B_{B}^{2}
$$

converges. Recall again that we are assuming that perturbation theory is valid in the asymptotic IR. Of course, there are theories (like QCD) where the couplings get large in the IR, invalidating the use of perturbation theory in the IR limit. We cannot draw any conclusions about such theories. But for theories that remain perturbative in the IR limit, we have shown that $T \rightarrow 0$ as an operator statement as $s \rightarrow 0$. By definition, this means that the theory is conformal in the IR.

Similar arguments can be made for the UV limit. These show that the UV limit is conformal if it is described in perturbation theory. Note that an implicit assumption of this argument is that the asymptotics is governed by a massless theory with a fixed Lagrangian, which does not allow theories with infinite numbers of massive particles coming in at higher and higher scales. Such theories are of course perfectly physical, so our constraint here is less general.

\subsection{Constraints from $J=0$}

Now let us consider the full sum over states Eq. (3.10), focusing in particular on the $J=0$ partial wave previously omitted. We must then consider contributions from the matrix element of $T\left(p_{1}+p_{1}\right)$ as well as $T\left(p_{1}\right) T\left(p_{1}\right)$. In perturbation theory, we expect the $T\left(p_{1}+p_{1}\right)$ terms to dominate because each derivative with respect to $\tau$ is proportional to a beta function. This is indeed correct, but the argument is 
somewhat subtle because we must exclude the possibility that the contact term in the $T\left(p_{1}\right) T\left(p_{2}\right)$ term has a contribution that is linear in beta functions. In $\S 3.5$ we will exclude this possibility using dimensional regularization, and in $\S 3.6$ we will derive an equivalent result from Wess-Zumino consistency conditions.

We will proceed assuming that the $T\left(p_{1}+p_{2}\right)$ term dominates in Eq. (3.10). This term can be written

$$
\begin{aligned}
s^{2} \operatorname{Im} \alpha(s) & =\operatorname{Im}\langle T(p) T(-p)\rangle \\
& =\sum_{A, B} B_{A}(s) B_{B}(s) \operatorname{Im}\left\langle\mathcal{O}^{A}(p) \mathcal{O}^{B}(-p)\right\rangle
\end{aligned}
$$

where $p^{2}=s$. For a perturbative theory the two-point function can be approximated by free field theory,

$$
\left\langle\mathcal{O}^{A}(p) \mathcal{O}^{B}(-p)\right\rangle=i c_{A} \delta^{A B} s^{2} \ln \left(-\frac{s+i \epsilon}{\mu^{2}}\right) .
$$

Eq. (3.4) then becomes

$$
r \partial_{r} \bar{\alpha}(r)=-2 \sum_{A} c_{A} B_{A}^{2},
$$

up to terms higher order in the beta functions, where $c_{A}$ are coefficients that are positive by unitarity. Specifically, for

$$
\begin{aligned}
\mathcal{O}^{1} & =\frac{1}{4 !} \Phi^{4}, & c_{1} & =\frac{1}{2^{10}(4 !)^{2} \pi^{6}}, \\
\mathcal{O}^{2} & =\Phi \bar{\Psi} \Psi, & c_{2} & =\frac{1}{2^{4} 4 ! \pi^{4}}, \\
\mathcal{O}^{3} & =F_{\mu \nu}^{2} / 4 g^{4}, & c_{3} & =\frac{1}{2^{5} \pi^{2} g^{4}},
\end{aligned}
$$

where $\Psi$ is a Dirac fermion. This implies that the flow of $\bar{\alpha} \sigma$ is monotonic. Because $\bar{\alpha}$ coincides with $a$ at conformal fixed points, this immediately gives a perturbative proof of the $a$-theorem. ${ }^{9}$ A very similar differential equation was obtained in Refs. $[7,8,9]$, as explained below in $\S 3.5$.

Using the same arguments given in $§ 3.2$, we can conclude that the integral

$$
\int \frac{d s}{s} B_{A}^{2}
$$

\footnotetext{
${ }^{9}$ In version 1 of this paper, Eq. (3.21) was interpreted as an RG equation for the coefficient $a$ of the WZ term. It was pointed out in Ref. [6] that this interpretation is not correct. However, the conclusions we draw from monotonicity of $\bar{\alpha}$ are not affected by this.
} 
converges both in the UV and the IR, provided the couplings remain perturbative. This conclusion is somewhat stronger than Eq. (3.18) derived previously.

\subsection{B versus $\beta$}

In this section we explain the distinction between $\beta_{A}$ and $B_{A}$ in Eq. (3.11) and comment on the significance of limit cycles as found in Refs. [3, 4]. To illustrate the basic idea we focus on general $\Phi^{4}$ theory with $N$ flavors whose renormalized Lagrangian (in flat space) is

$$
\mathcal{L}=\frac{1}{2} \partial_{\mu} \Phi_{i} \partial^{\mu} \Phi_{i}+\frac{\lambda_{i j k \ell}}{4 !} \Phi_{i} \Phi_{j} \Phi_{k} \Phi_{\ell}
$$

The $\beta$-functions and the anomalous dimensions of the fields are defined by expanding the trace of the canonical energy momentum tensor $T_{\mu}^{\mu}$ in a complete basis of renormalized composite operators. In the above theory, generalizing for instance Ref. [14], and indicating by $[\mathcal{O}]$ the renormalized operator one can write

$$
T=\beta_{i j k \ell}\left[\mathcal{O}_{i j k \ell}\right]+N_{i j} \partial_{\mu}\left[J_{i j}^{\mu}\right]+\Gamma_{i j}\left[\phi_{j} E_{i}\right]+a_{i j} \square\left[\phi_{i} \phi_{j}\right]
$$

where $\mathcal{O}_{i j k \ell}=\frac{1}{4 !} \Phi_{i} \Phi_{j} \Phi_{k} \Phi_{\ell}, J_{i j}^{\mu}$ is the flavor current, and $E_{i}=\delta S / \delta \Phi_{i}$ is the equation of motion operator. The coefficient of the third term defines the dimension matrix: $\Gamma_{i j}=\delta_{i j}+\gamma_{i j}$. Notice that the second term is absent in the case of the single flavor $\Phi^{4}$ theory of Ref. [14], but is generally expected by power counting: $N_{i j}$ is an antisymmetric $O(N)$ tensor covariantly constructed from the coupling tensor $\lambda_{i j k \ell}$. The last term can be improved away and plays no role in this discussion and in dilaton amplitudes. Note that the response of the theory to a global scale transformation is given by an insertion of $\int d^{4} x T(x)$, in which the second and fourth terms in Eq. (3.25) drop out because they are total derivatives. This gives the Callan-Symanzik equations for the theory.

The local Eq. (3.25) is what matters to describe the effect of conformal transformations, i.e. local dilations. However there is an inherent ambiguity in the definition of $\beta, N, \Gamma$. This is because the Ward identity for the broken flavor symmetry implies a linear relation among the corresponding three operators in Eq. (3.25). Given any $S$, generator of $O(n)$, the Ward identity states

$$
(S \cdot \lambda)_{i j k \ell}\left[\mathcal{O}_{i j k l}\right]+S_{i j}\left(\left[\phi_{j} E_{i}\right]+\partial_{\mu}\left[J_{i j}^{\mu}\right]\right)=0
$$

which added to Eq. (3.25) implies invariance under the reparametrization

$$
\beta_{i j k \ell} \rightarrow \beta_{i j k \ell}+(S \cdot \lambda)_{i j k \ell}, \quad \Gamma_{i j} \rightarrow \Gamma_{i j}+S_{i j}, \quad N_{i j} \rightarrow N_{i j}+S_{i j}
$$


Working in different renormalization schemes will in general lead to coefficients that differ by the above reparametrization. This complete freedom was not discussed in Ref. [9]: in Eq. (3.46) of that paper the ambiguity is limited to the case $S \propto \beta .^{10}$ The freedom in Eq. (3.27) corresponds to the freedom in choosing the RG flow coefficients discussed for the case of limit cycles in the Appendix.

A convenient way to "fix the gauge" is to choose $S=-N$ so that the coefficient of the current in Eq. (3.25) vanishes:

$$
T=\frac{B_{i j k \ell}}{4 !}\left[\mathcal{O}_{i j k \ell}\right]+\Delta_{i j}\left[\phi_{j} E_{i}\right]+a_{i j} \square\left[\phi_{i} \phi_{j}\right]
$$

where $B_{i j k \ell}=\beta_{i j k \ell}-(N \cdot \lambda)_{i j k \ell}$ and $\Delta_{i j}=\Gamma_{i j}-N_{i j}$. According to the result proven in the previous section, and further developed in the following sections, the only possible UV and IR asymptotics has $B=0$, corresponding to CFTs. In those asymptotic CFTs the eigenvalues of $\Delta_{i j}$ give the scaling dimensions of the elementary fields. From the general theory of the unitary representations of $S O(4,2)[15]$ these eigenvalues should all be real and $\geq 1$.

This discussion also clarifies the significance of limit cycles with $B=0$, such as those discussed in Ref. [4]. In such theories there exists a "gauge" for $\beta, N, \Gamma$ where $\beta=0$ and $N=0$. This corresponds to a scheme where the couplings are constant on the $\mathrm{RG}$ flow. In a general gauge one would have $\beta=(X \cdot \lambda)$ with $X$ a constant matrix: for these other choices the coupling would describe a cycle. It is however clear from the discussion that this cycling does not have a reparameterization-invariant meaning.

In the appendix we consider theories with limit cycles that may or may not have $B=0$. We show that these theories are also equivalent to theories with fixed points due to the same ambiguity.

\subsection{Dilaton Couplings in Dimensional Regularization}

We will now make the above discussion more explicit by working in dimensional regularization and defining composite operators by differentiation with respect to external sources, position dependent couplings and flavor gauge fields. The discussion in this section is largely extracted from Ref. [8]. Moreover, since our final goal are

\footnotetext{
${ }^{10}$ In Ref. [13] we will show how precisely the full ambiguity, with general $S$, arises when defining the $\mathrm{RG}$ flow in dimensional regularization. The basic point is that, given a theory renormalized with a well-defined pole subtraction procedure, there exists a family of possible choices of the RG flow coefficients, parametrized by Eq. (3.27), all describing the momentum evolution of the same correlators.
} 
the amplitudes between on-shell dilaton and on-shell fields, the equations of motion and the improvement terms will not play any role.

Consider the dimensionally regularized Lagrangian

$$
\begin{aligned}
\mathcal{L}_{0}=\sqrt{-g}\left[\frac{1}{2} g^{\mu \nu} D_{\mu} \hat{\Phi}_{0}^{i} D_{\nu} \hat{\Phi}_{0}^{i}-\frac{\lambda_{0 i j k l}}{4 !} \hat{\Phi}_{0}^{i} \hat{\Phi}_{0}^{j} \hat{\Phi}_{0}^{k} \hat{\Phi}_{0}^{l}\right. \\
\left.+N_{0 m n}^{i j k l} g^{\mu \nu} D_{\mu} \lambda_{0 i j k l} \Phi^{m} \stackrel{\leftrightarrow}{D}_{\nu} \Phi^{n}+\cdots\right]
\end{aligned}
$$

where

$$
D_{\mu} \hat{\Phi}^{i}=\partial_{\mu}-\left(A_{\mu}\right)^{i}{ }_{j} \hat{\Phi}^{j}
$$

is the flavor gauge covariant derivative. Note that the kinetic term is chosen to be canonical and therefore invariant under the flavor gauge group, which is $O(N)$ in this case. Now $\lambda_{0}$ as well as $g_{\mu \nu}$ are functions of $x$, and the last term is required by renormalizability in the theory with position-dependent couplings. We are dropping terms that do not matter for getting the coupling of an on-shell dilaton to the QFT, including all curvature terms and higher derivatives of the coupling. For a discussion of these terms, see Ref. [8]. Then for $g_{\mu \nu}=e^{-2 \tau} \eta_{\mu \nu}$ and defining $\Phi_{0}=e^{(2-d) \tau / 2} \hat{\Phi}_{0}$, this becomes

$$
\mathcal{L}_{0}=\frac{1}{2} D_{\mu} \Phi_{0}^{i} D_{\mu} \Phi_{0}^{i}-e^{-\epsilon \tau} \frac{\lambda_{0 i j k l}}{4 !} \Phi_{0}^{i} \Phi_{0}^{j} \Phi_{0}^{k} \Phi_{0}^{l}+N_{0 m n}^{i j k l} D^{\mu} \lambda_{0 i j k l} \Phi_{0}^{m} \stackrel{\leftrightarrow}{D}{ }_{\mu} \Phi_{0}^{n}
$$

There is no $\partial \tau$ from the last term because of antisymmetry in $m n$.

Note that with the couplings defined as flavor spurions, the flavor symmetry is exact, and so the flavor gauge fields do not need to be renormalized. $N_{0}$ can be chosen to have no finite part, so it is a sum of poles in $1 / \epsilon$. We then write $\mathcal{L}_{0}=\mathcal{L}_{0}^{(0)}+\mathcal{L}_{0}^{(2)}+\cdots$ where $\mathcal{L}_{0}^{(n)}$ is of order $\tau^{n}$. The linear coupling of $\tau$ is then

$$
\begin{aligned}
\mathcal{L}_{0}^{(1)} & =\epsilon \tau \frac{\lambda_{0 i j k l}}{4 !} \Phi_{0}^{i} \Phi_{0}^{j} \Phi_{0}^{k} \Phi_{0}^{l} \\
& =-\epsilon \tau \lambda_{0 i j k l} \frac{\delta S_{0}}{\delta \lambda_{0 i j k l}}+\epsilon \tau \lambda_{0 i j k l} N_{0 m n}^{i j k l} \partial_{\mu} \Phi_{0}^{m} \partial_{\mu} \Phi_{0}^{n} \\
& =-\epsilon \tau \lambda_{0 i j k l} \frac{\partial \lambda_{i^{\prime} j^{\prime} k^{\prime} l^{\prime}}}{\partial \lambda_{0 i j k l}} \frac{\delta S_{0}}{\delta \lambda_{i^{\prime} j^{\prime} k^{\prime} l^{\prime}}}+\epsilon \tau \lambda_{0 i j k l} N_{0 m n}^{i j k l} \partial_{\mu} \frac{\delta S_{0}}{\delta \bar{A}_{m n}^{\mu}}
\end{aligned}
$$

where $\bar{A}_{\mu m n}$ is defined by

$$
\bar{A}_{\mu m n}=A_{\mu m n}+N_{0 i j k l}^{m n}\left(A_{\mu} \lambda_{0}\right)^{i j k l} .
$$


The second term comes from the covariant derivative acting on $\lambda_{0}$ in the Lagrangian. We can simplify the first term in Eq. (3.32) using the dimensional renormalization relation

$$
\epsilon \lambda_{0 i j k l}+\frac{\partial \lambda_{0 i j k l}}{\partial \lambda_{i^{\prime} j^{\prime} k^{\prime} l^{\prime}}} \beta_{i^{\prime} j^{\prime} k^{\prime} l^{\prime}}=0,
$$

and it is then explicitly finite. Since the total $\tau$ coupling is renormalized, the second term must also be finite. By expressing it in terms of $\delta / \delta A_{m n}^{\mu}$, which is also finite by construction, the only surviving term in the coefficient must be the one proportional to $\epsilon^{0}$ term. Because $N_{0}$ is a series of pure poles, the $\epsilon^{0}$ term is just given by the residue of the single-pole term in $N_{0} \cdot{ }^{11}$ Therefore, the linear coupling of $\tau$ is

$$
\mathcal{L}_{0}^{(1)}=\tau \beta_{i^{\prime} j^{\prime} k^{\prime} l^{\prime}} \frac{\delta S_{0}}{\delta \lambda_{i^{\prime} j^{\prime} k^{\prime} l^{\prime}}}+\tau \lambda_{i j k l} N_{1 m n}^{i j k l} \partial_{\mu} \frac{\delta S_{0}}{\delta A_{m n}^{\mu}}
$$

where $N_{1}$ is the residue of the $1 / \epsilon$ pole in $N_{0}$, which is a finite quantity. The second term can be simplified via the equation of motion [8] (in the notation of that paper $\left.S=\lambda N_{1}\right)$. We then obtain

$$
\mathcal{L}_{0}^{(1)}=\tau B_{i^{\prime} j^{\prime} k^{\prime} l^{\prime}} \frac{\delta \mathcal{L}_{0}}{\delta \lambda_{i^{\prime} j^{\prime} k^{\prime} l^{\prime}}} .
$$

Now consider the couplings of order $\tau^{2}$. From Eq. (3.31),

$$
\mathcal{L}_{0}^{(2)}=\frac{1}{2} \epsilon \mathcal{L}_{0}^{(1)}
$$

However, since $\mathcal{L}_{0}^{(1)}$ is finite, the quadratic coupling must vanish at $d=4$. A local $\tau^{2}$ coupling does arise from a contact term of order $B \partial_{\lambda} B$ appearing from double insertion of the linear coupling to $\tau$ [13]. However, this term is parametrically suppressed compared to the $O(B)$ term arising from the nonlinear relation between $\tau$ and $\varphi$, which was used in the argument of $\S 3.3$.

\subsection{Constraints from Wess-Zumino Consistency}

The discussion in this section is largely extracted from Ref. [9]. A flow equation of the same form as Eq. (3.21) can be obtained by application of the WZ consistency relations. Consider a renormalized generating functional $W\left[g_{\mu \nu}, \lambda_{A}, A^{\mu a}\right]$. Here $\lambda_{A}$ are a complete set of couplings for dimension- 4 scalars and $A^{\mu a}$ are a complete set of

\footnotetext{
${ }^{11}$ Note that, via eq. (3.33), the $1 / \epsilon$ pole in $N_{0}$ also gives a contribution to the anomalous dimension of the flavor current.
} 
flavor gauge fields, that is, couplings for dimension-3 vector operators. The couplings $\lambda_{A}$ as well as $g_{\mu \nu}$ and $A_{\mu a}$ are allowed to depend on $x$. Various dimension-2 scalars are also needed, but do not enter into the following discussion. The couplings of $A_{\mu a}$ are restricted by background gauge invariance and so it appears in covariant derivatives and field strengths, e.g. $D_{\mu} \lambda_{A}=\partial_{\mu} \lambda_{A}+A_{\mu a}\left(T_{a}\right)_{A}{ }^{B} \lambda_{B}$, where $T_{a}$ are the generators of the flavor group.

The Weyl variation of $W$ can be written in terms of a sum of all local terms, $\delta_{x} W=D(x)$, where $D(x)$ includes all possible dimension-4 functions of the sources, including gradients of couplings and curvatures. The Weyl variation operator is

$$
\delta_{x}=\frac{\delta}{\delta \tau(x)}-\beta_{A} \frac{\delta}{\delta \lambda_{A}(x)}-\rho_{a}^{A} D_{\mu} \lambda_{A} \frac{\delta}{\delta A_{\mu a}(x)}-D_{\mu}\left(S_{a} \frac{\delta}{\delta A_{\mu a}(x)}\right)
$$

Using background gauge invariance, we can collect the last term into the others:

$$
\delta_{x}=\frac{\delta}{\delta \tau(x)}-B_{A} \frac{\delta}{\delta \lambda_{A}(x)}-P_{a}^{A} D_{\mu} \lambda_{A} \frac{\delta}{\delta A_{\mu a}(x)},
$$

with $B_{A}=\beta_{A}-(S \lambda)_{A}$ and $P_{a}^{A}\left(D_{\mu} \lambda\right)_{A}=\rho_{a}^{A}\left(D_{\mu} \lambda\right)_{A}+\left(D_{\mu} S\right)_{a}$. That is, $B$ is the total Weyl variation of the dimension- 4 couplings, and $P$ is the total Weyl derivative of the vector couplings. Thus the condition for conformal invariance is $B=0$.

The Wess-Zumino consistency condition is

$$
\left[\delta_{x}, \delta_{y}\right] W=\delta_{x} D(y)-\delta_{y} D(x)
$$

To derive the flow equation, we only need terms proportional to the Einstein tensor, which arise from

$$
D(x)=\beta_{(b)} E_{4}+\frac{1}{2} G^{\mu \nu} \chi_{(\lambda)}^{A B} D_{\mu} \lambda_{A} D_{\nu} \lambda_{B}+G^{\mu \nu} D_{\mu}\left(w^{A} D_{\nu} \lambda_{A}\right)+\ldots
$$

Picking out terms proportional to $G^{\mu \nu} D_{\mu} \lambda_{A} \delta^{\prime}(x-y)$, the consistency relation becomes

$$
8 \frac{\partial \beta_{(b)}}{\partial \lambda_{A}}=\chi_{(\lambda)}^{A B} B_{B}-\frac{\partial w^{A}}{\partial \lambda_{B}} B_{B}-\frac{\partial B_{B}}{\partial \lambda_{A}} w^{B}+P_{a}^{A}\left(T_{a} \lambda\right)_{B} w^{B} .
$$

Now, contracting with $B_{A}$, defining $\tilde{B}_{(b)}=\beta_{(b)}+\frac{1}{8} w^{A} B_{A}$, and using $B_{A} P^{A}=0$ (from the $\delta / \delta A$ term in the Wess-Zumino condition) gives $[9,6]$

$$
8 B^{A} \partial_{A} \tilde{B}_{(b)}=\chi_{(\lambda)}^{A B} B_{A} B_{B}
$$

This gives the flow equation

$$
\mu \frac{d \tilde{B}_{(b)}}{d \mu}=B_{A} \frac{\partial \tilde{B}_{(b)}}{\partial \lambda_{A}}=\beta_{A} \frac{\partial \tilde{B}_{(b)}}{\partial \lambda_{A}}=\frac{1}{8} \chi_{(\lambda)}^{A B} B_{A} B_{B} .
$$


(Note that $B$ and $\beta$ give the same flow here because $\tilde{B}_{(b)}$ is invariant under field rotations).

In perturbation theory, $\chi_{(\lambda)}^{A B}$ is positive, and the parallel between the flow Eq. (3.44) of $\tilde{B}_{(b)}$ and the flow Eq. (3.21) of $\bar{\alpha}$ is evident. In particular, each is stationary only at conformal points, and so any perturbative flow must approach a conformal theory in the UV and the IR. ${ }^{12}$

\section{Scale Versus Conformal Invariance}

In this section, we consider in more generality the question of whether scale invariance implies conformal invariance, without assuming the validity of perturbation theory. In $2 \mathrm{D}$ there is a rigorous argument that scale invariance implies conformal invariance [17] based on the Zamolodchikov $c$-theorem [18]. Given that we now have a (nonperturbative) proof of the $a$-theorem in $4 \mathrm{D}$, it is natural to ask whether we can give a similar proof for 4D theories. This was shown at the classical level in Ref. [19], but of course quantum effects play an essential role.

The results of $\S 3$ already show that scale invariance implies conformal invariance for weakly-coupled flows. In particular, for nonconformal scale invariant theories (SFTs) the dilaton does not decouple; see also the recent discussion [20]. For nonperturbative theories, we will show that scale invariance implies conformal invariance subject to a plausible technical assumption. Various aspects of our argument will also be checked in perturbative examples that have some but not all of the features of unitary SFTs.

\subsection{Generalities}

Consider a possible theory that is scale invariant but not conformally invariant, a SFT. It was shown by Wess [21] that the most general conserved scale current has the form

$$
S^{\mu}=T_{\nu}^{\mu} x^{\nu}+V^{\mu}
$$

where $T_{\mu}^{\mu}$ is the stress-energy tensor and $V_{\mu}$ is called the virial current. (We will not consider scale invariant theories that do not have a local scale current.) Conservation of the scale current then implies

$$
0=\partial_{\mu} S^{\mu}=T+\partial_{\mu} V^{\mu}
$$

\footnotetext{
${ }^{12}$ As will be shown in Ref. [13], $\chi_{(\lambda)}^{A B}$ is indeed positive definite for perturbations around any conformal fixed point.
} 
The theory is conformal if $T \equiv 0$, so we see that we can get a nontrivial SFT only if $\partial_{\mu} V^{\mu} \neq 0$.

We can write the Ward identities for scale invariance in a convenient form by introducing a source $C_{\mu}$ for the virial current as well as using $g_{\mu \nu}$ as a source for $T^{\mu \nu}$. For local scale transformations generated by $\sigma(x)$ we then have

$$
W_{\mathrm{SFT}}\left[e^{2 \sigma} g_{\mu \nu}, C_{\mu}+\partial_{\mu} \sigma\right]=W_{\mathrm{SFT}}\left[g_{\mu \nu}, C_{\mu}\right]+S_{\mathrm{WZ}}\left[\sigma ; g_{\mu \nu}, C_{\mu}\right]
$$

where $S_{\mathrm{WZ}}$ is an anomaly term. The anomaly term is local and must satisfy the Wess-Zumino consistency conditions, which enforce that local scale transformations are Abelian. In addition, the WZ term cannot contain any dimensionful parameters.

The most general WZ term satisfying these constraints can be readily found. It includes the $a$ and $c$ terms from conformal field theories, which do not depend on $C_{\mu}$. There are only two additional allowed terms

$$
\Delta S_{\mathrm{WZ}}=\int d^{4} x \sqrt{-g} \sigma\left[e \mathcal{O}_{e}+f \mathcal{O}_{f}\right]
$$

where

$$
\begin{aligned}
& \mathcal{O}_{e}=\frac{1}{12} R^{2}(g)+R(g) \nabla \cdot C-R(g) C^{2}+3(\nabla \cdot C)^{2}-6 C^{2} \nabla \cdot C+3 C^{4}, \\
& \mathcal{O}_{f}=C^{\mu \nu} C_{\mu \nu}
\end{aligned}
$$

where $C_{\mu \nu}=\partial_{\mu} C_{\nu}-\partial_{\nu} C_{\mu}$. The $e$ term will play an important role in our arguments below.

\subsection{Non-perturbative Argument}

We again consider the amplitude $A(s)$ defined above. That is, we consider background fields

$$
\hat{g}_{\mu \nu}=\left(1+\frac{\varphi}{f}\right)^{2} \eta_{\mu \nu}, \quad C_{\mu} \equiv 0,
$$

and define "dilaton amplitudes" by differentiating $W\left[\hat{g}_{\mu \nu}, 0\right]$ with respect to $\varphi$, taking the forward limit, and imposing the "on-shell" condition $\square \varphi=0$.

We can find the exact form of this amplitude in an SFT using the Ward identity Eq. (4.3) with $g_{\mu \nu}=\hat{g}_{\mu \nu}, C_{\mu}=0$, and $\sigma=$ constant. The anomaly term in Eq. (4.3) does not contribute to the transformation of $A(s)$ : the a term vanishes (for $\sigma=$ constant) because $E_{4}(g)$ is a total derivative, the $c$ term vanishes for conformally flat backgrounds, the $e$ and $f$ terms vanishes because of the on-shell condition $R(g)=0$ 
and because we are considering backgrounds with $C_{\mu}=0$. The fact that there is no anomalous scaling of the amplitude is equivalent to the statement that there is no counterterm for this amplitude that can have a logarithmic dependence on the cutoff.

Because there is no anomalous contribution to the amplitude $A(s)$, it has the exact form dictated by naïve scale invariance, namely

$$
A(s)=\frac{\alpha s^{2}}{f^{4}},
$$

with $\alpha=$ constant. (Here we are assuming that the vacuum is scale invariant, so this does not hold for nonlinearly realized scale invariance. An example of this kind will be discussed in $\S 4.3$ below.) This has no imaginary part, which has strong implications in a unitary theory. Unitarity implies that the imaginary part of $A(s)$ is positive, and in fact can be written as a sum over a complete set of state states, with each state giving a positive contribution. Therefore, the only way we can have a vanishing imaginary part is for each term to vanish individually. We must therefore have

$$
\mathcal{O}\left(p_{1}, p_{2}\right)|0\rangle=0
$$

where

$$
\mathcal{O}\left(p_{1}, p_{2}\right)=T\left(p_{1}\right) T\left(p_{2}\right)+T\left(p_{1}+p_{2}\right)
$$

(Here the trace of the stress tensor is defined as in Eq. (3.8) above.) If Eq. (4.9) were to hold for arbitrary momenta $p_{1,2}$ then we could immediately conclude that $\mathcal{O}\left(p_{1}, p_{2}\right) \equiv 0$ as an operator statement. In position space, this is the statement that

$$
\mathrm{T}\left\{T\left(x_{1}\right) T\left(x_{2}\right)\right\}=-\delta^{4}\left(x_{1}-x_{2}\right) T\left(x_{1}\right)
$$

which can only hold in a theory where $T$ is a trivial operator. However, our arguments hold only for on-shell dilaton amplitudes with $p_{1,2}^{2}=0$. We cannot extend our arguments to off-shell amplitudes because then the $e$ anomaly term Eq. (4.5) contributes to the scale transformation of $A(s)$, allowing a log term in $A(s)$. This corresponds to a logarithmic renormalization of an $R^{2}$ counterterm. ${ }^{13}$

It is interesting to contrast this situation with the $d=2 \operatorname{argument}[17,18]$. In that case, the vanishing of the $T$ 2-point function immediately implies that $T$ must vanish (with no on-shell condition).

\footnotetext{
${ }^{13}$ As discussed in $\$ 2.3$, a logarithmic renormalization of $R^{2}$ arises in CFTs deformed by a term $\mathcal{R O}$ where $\mathcal{O}$ has dimension $2\left(e . g . \mathcal{O}=\Phi^{2}\right.$ in a theory of a free scalar). This is not surprising since this is an explicit marginal breaking of conformal invariance.
} 
One scenario where the amplitude $A(s)$ has no imaginary part in a SFT with a nontrivial operator $T$ is to have a factorized $T$ amplitude:

$$
\begin{aligned}
& \left\langle 0\left|\mathrm{~T}\left\{T\left(x_{1}\right) T\left(x_{2}\right) T\left(x_{3}\right) T\left(x_{4}\right)\right\}\right| 0\right\rangle \\
& =\frac{\text { constant }}{\left(x_{1}-x_{2}\right)^{8}\left(x_{3}-x_{4}\right)^{8}}+\text { permutations }+ \text { contact terms } .
\end{aligned}
$$

The nonlocal part of this amplitude contributes only to forward scattering, and therefore does not contribute to $\operatorname{Im} A$. Then $\operatorname{Im} A$ is completely local, and this local term can vanish. (Note that large- $N$ theories do not provide the necessary factorized form. The leading contribution in the large- $N$ limit is disconnected, connected amplitudes remain at subleading order in the $1 / N$ expansion.) The disconnected amplitude Eq. (4.12) implies a TT operator product expansion (OPE) that contains only the unit operator and nontrivial operators starting at dimension 8. Even if the theory satisfies all this, $C=0$ requires a cancellation between the (presumably infinitely many) operators appearing in the TT OPE and the TT contribution in Eq. (3.7). In a CFT we could conclude that $T$ appears in the TT OPE from the symmetry of the OPE coefficients, but it is not clear that this is so in an SFT, as the position dependence of the three-point function is undetermined. We regard this exception as implausible, and likely to be ruled out in the future.

\subsection{Examples}

For a nonunitary theory, a nonzero $T$ can still give rise to a vanishing $C$ by cancellation between positive and negative contributions. Our argument, that divergent renormalization of $a$ is inconsistent, requires that such cancellation occur in any nonunitary SFT. A massless vector field without gauge invariance provides a such a theory, in any dimension. This was first noted by Coleman and Jackiw [22] in $d=4$. It was studied in detail in $d=2$ by Riva and Cardy (RC) [23], who also observed that it has a Euclidean interpretation as the theory of elasticity.

The action is written in terms of a displacement field $u_{\mu}(x)$. Following $\mathrm{RC}$ we start with a Euclidean action, which is the physically relevant signature for elasticity,

$$
S=\int d^{4} x \sqrt{-g}\left(\frac{1}{4} F_{\mu \nu} F^{\mu \nu}+\frac{h}{2}\left(\nabla_{\mu} u^{\mu}\right)^{2}\right) .
$$

Here $F_{\mu \nu}=\partial_{\mu} u_{\nu}-\partial_{\nu} u_{\mu}$. (In the notation of RC, $g=1$ and $k=h-2$.)

Scale invariance is manifest, with $u_{\mu}$ having dimension 1 . Consider now the metric $g_{\mu \nu}=e^{-2 \tau} \delta_{\mu \nu}$. The Maxwell-like term is conformally invariant in $d=4$. Using 
$\nabla_{\mu} u^{\mu}=\sqrt{g}^{-1} \partial_{\mu}\left(\sqrt{g} g^{\mu \nu} u_{\nu}\right)=e^{4 \tau} \partial_{\mu}\left(-e^{2 \tau} u_{\mu}\right)$, the coupling the dilaton is

$$
2 h \partial_{\mu} u^{\mu} u^{\nu} \partial_{\nu} \varphi-2 h \partial_{\mu} u^{\mu} u^{\nu} \varphi \partial_{\nu} \varphi+2 h\left(u^{\mu} \partial_{\mu} \varphi\right)^{2}+O\left(\varphi^{3}\right)
$$

and so the theory is not conformally invariant. We can regulate this theory using Pauli-Villars in the background $\hat{g}_{\mu \nu}$. Our arguments then imply that there should be no running of $a$. This is true, but it is nontrivial, and provides a test of our reasoning.

If we rotate to Lorentzian signature, these couplings give a $\varphi \varphi \rightarrow u u$ scattering amplitude, which has a contact piece from the $\varphi^{2}$ terms in the interaction (4.14) and pole and contact pieces from second order in the $\varphi$ term. If the total cross section obtained by 'squaring' and integrating over phase space were nonzero, it would be proportional to $s^{2}$ from the scaling of the vertices, and the dispersive argument in Fig. 1 would imply a logarithmic divergence of $a$; we have argued this to be inconsistent. In fact, direct calculation shows that there is no logarithmic divergence, and so no imaginary part in the forward amplitude. Individual graphs are nontrivial functions of $h$, but the sum vanishes identically. This cancellation provides a satisfying check of the general argument. Of course, the vanishing of the total cross section is possible only because of the nonunitarity of the theory. In a unitary theory the differential cross section would have to vanish identically.

The calculation of the logarithmic divergence is uninstructive, but we can give an independent indirect argument for it, exploiting the resemblance between $u_{\mu}$ and a gauge field. First, we can write the theory in a gauge invariant way by introducing a Goldstone field $\pi$,

$$
S=\int d^{4} x \sqrt{-g}\left(\frac{1}{4} F_{\mu \nu} F^{\mu \nu}+\left[\nabla^{\mu}\left(\partial_{\mu} \pi+h^{1 / 2} u^{\mu}\right)\right]^{2}\right) .
$$

In the gauge $\pi=0$ this reduces to the earlier action. Now, at $h=0$ this becomes

$$
S=\int d^{4} x \sqrt{-g}\left(\frac{1}{4} F_{\mu \nu} F^{\mu \nu}+(\square \pi)^{2}\right) .
$$

Both terms are now conformally invariant and so the on-shell dilaton coupling vanishes. In particular, by adding improvement terms $\square \pi \rightarrow(\square+R / 6) \pi$ we can make the action fully Weyl-invariant.

This implies the vanishing of the cross section at $h=0$. To extend this, we look at the action in a different way. The $h\left(\nabla_{\mu} u^{\mu}\right)^{2} / 2$ term can be interpreted as a covariant gauge-fixing term. We might then conclude that the dilaton decouples due to the conformal invariance of the pure Maxwell theory. However, we did not include the associated ghost determinant, which will have a nontrivial dependence on 
the metric. The ghost action is independent of $h$, and so we can argue from this that the renormalization of $a$ is independent of $h$, and so vanishes for all $h$.

Another potential counterexample noted in the classic literature [19] is the scalar Lagrangian

$$
\mathcal{L}=\frac{1}{2} \partial^{\mu} \hat{\Phi} \partial_{\mu} \hat{\Phi}+\frac{\left(\partial^{\mu} \hat{\Phi} \partial_{\mu} \hat{\Phi}\right)^{2}}{\hat{\Phi}^{4}} .
$$

This Lagrangian is manifestly scale invariant but not conformally invariant. It is nonrenormalizable, but in a state with $\langle\hat{\Phi}\rangle \neq 0$ we can still consider it as an effective field theory below the scale $\langle\hat{\Phi}\rangle$, with scale invariance nonlinearly realized in an expansion in inverse powers of $\langle\hat{\Phi}\rangle$. There is no symmetry forbidding a $\hat{\Phi}^{4}$ term, which would forces the ground state to be at $\langle\hat{\Phi}\rangle=0$, but this term can be tuned away. There are also an infinite number of additional terms with higher powers of derivatives that give higher order corrections in the low-energy expansion. These do not affect the arguments below.

As noted below Eq. (4.8), our general argument ruling out SFTs assumes that scale invariance is linearly realized, and so this theory does not conflict with those arguments. However, the argument that $a$ is not renormalized in a SFT applies to this theory, so we can check it. In flat spacetime, it is natural to expand

$$
\hat{\Phi}=v+\hat{\pi} .
$$

Setting $\hat{g}_{\mu \nu}=\Omega^{2} \eta_{\mu \nu}$ and

$$
\hat{\pi}=\Omega^{-1} \pi,
$$

the Lagrangian becomes

$$
\mathcal{L}=\frac{1}{2}(\partial \pi)^{2}+\frac{\left[\partial\left(\Omega^{-1} \pi\right)\right]^{4}}{\left(v+\Omega^{-1} \pi\right)^{4}} .
$$

where we set $\square \Omega=0$. Note that there is no mixing between the background dilaton and the dynamical dilaton field $\pi$. All interactions of the dilaton involve inverse powers of the symmetry breaking scale $v$, giving a cross section that scales as

$$
\sigma(\varphi \varphi \rightarrow \pi \pi) \sim \frac{s^{5}}{v^{8} f^{4}} .
$$

Higher-order interactions will give additional terms with additional positive powers of $s / v^{2}$. These give power law divergences in the dispersion integral, which just cancel the negative powers of $v$ from the cross section: there is no logarithmic dependence on the UV cutoff, consistent with our general arguments. 


\section{Conclusions}

We have shown that the method introduced in Refs. [1, 2], using properties of the $a$-anomaly to show irreversibility in the flow between 4D CFT's, can be extended to exclude a wide variety of other $4 \mathrm{D}$ flows. The key observation is that the total flow of the scattering amplitude $\alpha(s)$ defined in Eq. (1.3) must be finite. We can then show rigorously that the only IR or UV asymptotics that can be described in perturbation theory is that of a conformal field theory. This excludes theories with scale without conformal invariance, such as those proposed in Ref. [3]. We can extend this argument beyond perturbation theory to rule out scale without conformal invariance in theories that can be deformed to a CFT in the UV or IR at an adjustable scale. We can rule out general non-perturbative theories with scale but not conformal invariance subject to a technical assumption: that vanishing of the imaginary part of the amplitude Eq. (3.7) implies vanishing of $T_{\mu}^{\mu}$. This is quite plausible, as the imaginary part of the amplitude receives contributions of the same sign from all intermediate states, so all of these must vanish.

We have tested our argument against various possible counterexamples. The nonunitary theory of Riva and Cardy evades the argument by cancellation of positive and negative contributions, but confirms our key observation that the renormalization of $\alpha$ must be finite. Theories with nonlinearly realized scale invariance are not ruled out by our arguments, but the argument that $\alpha$ has no logarithmic dependence on the cutoff can be checked in these theories. Our results predict that the limit cycles discussed in Ref. [3] are conformal, as has been recognized by the authors [4].

Monotonicity of the RG flow has not been established aside from 2 and 4 dimensions, although various ideas are being explored. The apparent lesson from the known cases is that monotonicity arguments can be extended to exclude SFT's and other exotic RG flows, but that these are not immediate corollaries; rather, they use the same machinery in new ways.

Finally, any discussion of RG flows will have a geometric analog via holography. Refs. [24] investigate possible holographic realizations of theories with scale but not conformal invariance. These theories violate the null energy condition, strongly suggesting that they are unphysical. On the other hand, our arguments rely on the renormalization properties of the theory in some background state, and do not appear to require the stability of this state. The consistency of the holographic theories with scale and not conformal invariance and their relation to our work is an interesting subject for future investigation. 


\section{Acknowledgements}

We thank D. Shih for collaboration in the early stages of this work, and S. Dubovsky, J.-F. Fortin, B. Grinstein, B. Keren-Zur, J. Maldacena, H. Osborn, D. Pappadopulo, I. Rothstein, S. Rychkov, A. Stergiou, R. Sundrum, and A. Zaffaroni for discussions. MAL and RR thank the KITP Santa Barbara for hospitality during the initial stages of this work. This research was supported in part by the NSF under grants PHY0757035 and PHY11-25915, by the DOE under grant DE-FG02-91-ER40674 and by the Swiss National Science Foundation under grant 200021-125237.

\section{Appendix: Callan-Symanzik Equations, Flavor, and Limit Cycles}

In this appendix we show that limit cycles are completely equivalent to fixed points due to the inherent ambiguity in renormalizing theories with flavor. We will actually prove a slightly stronger result, namely that any $\mathrm{RG}$ flow that is equivalent to a scale-dependent flavor rotation is equivalent to a fixed point. The renormalization ambiguity described here for limit cycles is a special case of the one discussed in $\S 3.4$, and the precise connection will be made explicit in Ref. [13].

To illustrate our ideas, we consider $\Phi^{4}$ theory with $N$ scalar fields, with renormalized Lagrangian

$$
\mathcal{L}_{R}=\frac{1}{2} \partial \Phi^{i} \partial \Phi^{i}-\frac{\lambda_{i j k l}}{4 !} \Phi^{i} \Phi^{j} \Phi^{k} \Phi^{l}
$$

The renormalized Lagrangian Eq. (A.1) contains all the information that is needed to compute the amplitudes of the theory. The counterterms, and hence the bare couplings, are determined order-by-order in perturbation theory by requiring the cancelation of the $1 / \epsilon$ divergences that appear.

We now turn to the Callan-Symanzik equations of the theory, which we will see are ambiguous in theories with flavor. The Callan-Symanzik equations state that correlation functions of the fields are independent of $\mu$ up to a $\mu$-dependent rescaling

of the fields. We can express this as the requirement that the correlation functions of rescaled fields $\hat{\Phi}$ are independent of $\mu$. We write

$$
\Phi^{i}=\xi_{a}^{i} \hat{\Phi}^{a} .
$$

Note that we are free to redefine the fields $\hat{\Phi}^{a}$ by a $G L(N)$ transformation acting on the $a$ index, corresponding to an arbitrary field redefinition that is linear in the fields. It is conventional to choose $\xi^{a}{ }_{i}$ to be symmetric, but there is nothing that forbids 
a choice where $\xi_{a}^{i}$ has an antisymmetric part. We will see below that this freedom allows us to rewrite limit cycles as fixed point. The Callan-Symanzik equation is then

$$
\begin{aligned}
& \left(\frac{\partial}{\partial t}+\beta_{i j k l} \frac{\partial}{\partial \lambda_{i j k l}}\right)\left\langle\Phi^{i_{1}} \cdots \Phi^{i_{n}}\right\rangle \\
& \quad=\gamma_{k}^{i_{1}}\left\langle\Phi^{k} \Phi^{i_{2}} \cdots \Phi^{i_{n}}\right\rangle+\cdots+\gamma_{k}^{i_{n}}\left\langle\Phi^{i_{1}} \cdots \Phi^{i_{n-1}} \Phi^{k}\right\rangle .
\end{aligned}
$$

where

$$
\begin{aligned}
\frac{d}{d t} \lambda_{i j k l} & =\beta_{i j k l}(\lambda), \\
\frac{d}{d t} \xi^{i}{ }_{a} & =\gamma_{j}^{i}(\lambda) \xi^{j}{ }_{a} .
\end{aligned}
$$

The form of Eq. (A.5) and the fact that $\beta$ and $\gamma$ do not depend on $\xi$ can be understood from covariance under $G L(N)$.

We now consider RG flows in which RG flow is equivalent to a flavor rotation, i.e.

$$
\lambda(t)=R(t) * \bar{\lambda}
$$

where $R(t)$ is a scale-dependent $O(N)$ transformation and $\bar{\lambda}$ is a scale-independent renormalized coupling. We are using an abstract notation where $*$ denotes the $O(N)$ action in the appropriate representation. For example, Eq. (A.6) is short for

$$
\lambda_{i j k l}(t)=\bar{\lambda}_{i^{\prime} j^{\prime} k^{\prime} l^{\prime}}\left(R^{-1}(t)\right)^{i^{\prime}}{ }_{i}\left(R^{-1}(t)\right)^{j^{\prime}}{ }_{j}\left(R^{-1}(t)\right)^{k^{\prime}}{ }_{k}\left(R^{-1}(t)\right)^{l^{\prime}}{ }_{l} .
$$

If we make the redefinition of renormalized fields

$$
\Phi^{i}=R_{j}^{i}(t) \Phi^{\prime j}
$$

the renormalized Lagrangian becomes

$$
\mathcal{L}_{R}=\frac{1}{2} \partial \Phi^{\prime i} \partial \Phi^{i}-\frac{\bar{\lambda}_{i j k l}}{4 !} \Phi^{\prime i} \Phi^{\prime j} \Phi^{\prime k} \Phi^{\prime l}
$$

That is, the theory is equivalent to a fixed point theory. ${ }^{14}$ We emphasize that Eq. (A.8) is a completely finite field redefinition, and therefore there is no need to reconsider the renormalization of the theory in terms of the new fields.

\footnotetext{
${ }^{14}$ In more general flows it is also natural to make the field redefinition Eq. (A.8) where $R$ depends on $t$ via the renormalized couplings $\lambda(t)$. This changes the beta function, but it cannot relate a fixed point theory to one with nonzero beta functions.
} 
In fact, we can determine the explicit form of $R(t)$ as follows. Requiring that Eq. (A.6) solves the RG equations gives

$$
\frac{d}{d t}(R(t) * \bar{\lambda})=\beta(R(t) * \bar{\lambda})=R(t) * \beta(\bar{\lambda}),
$$

where we have used the flavor covariance of the $\beta$ function in the last step. This just means that the only violation of flavor symmetry comes from the couplings, which can be taken to be spurions for the flavor symmetry. We therefore have

$$
\left[R^{-1}(t) \frac{d}{d t} R(t)\right] * \bar{\lambda}=\beta(\bar{\lambda}) .
$$

Since the right-hand side is independent of $t$, the left-hand-side must be as well, which means that the scale-dependent $O(N)$ transformation must have the form

$$
R(t)=e^{-t X}
$$

for some fixed flavor generator $X$ constructed from the couplings $\bar{\lambda}$. This is the form of the RG cycles considered by Refs. [3, 4].

We can similarly analyze the RG equation for the wavefunction factors, Eq. (A.5). Covariance of the anomalous dimension function $\gamma$ implies that

$$
\frac{d}{d t} \xi_{a}^{i}=\left(e^{-t X}\right)_{i^{\prime}}^{i} \gamma^{i^{\prime}}{ }_{j^{\prime}}(\bar{\lambda})\left(e^{t X}\right)^{j^{\prime}}{ }_{j} \xi^{j}{ }_{a}
$$

This is solved by

$$
\xi(t)=e^{-t X} e^{t(X+\bar{\gamma})}
$$

where $\bar{\gamma}=\gamma(\bar{\lambda})$. This solution is not unique because we can multiply on the right by an arbitrary $t$-independent linear transformation. This solution therefore makes a canonical identification between $a$ and $i$ indices.

In terms of the new fields $\Phi^{\prime}$ given by Eq. (A.8), we have

$$
\xi^{\prime}=R^{-1} \xi=e^{t(X+\bar{\gamma})} .
$$

The renormalized Lagrangian is therefore invariant under scale transformations of the form

$$
\Phi^{\prime}(x) \mapsto e^{\Delta t} \Phi^{\prime}\left(e^{t} x\right) .
$$

Here

$$
\Delta=\Delta_{0}+\bar{\gamma}+X
$$


where $\Delta_{0}$ is the canonical dimension of the fields ( 1 for scalars $\frac{3}{2}$ for fermions). Note that gauge fields do not have independent wavefunction renormalization factors. For example, the 2-point function of these fields is given by

$$
\left\langle\Phi^{\prime i}(x) \Phi^{\prime j}(0)\right\rangle=\frac{1}{x^{\Delta}} C\left(\frac{1}{x^{\Delta}}\right)^{T} .
$$

where $C^{i j}=C^{j i}$ are constants. We see that in terms of the fields $\Phi^{\prime}$ the scale invariance is manifest. According to the main result of this paper, scale invariance should extend to the full conformal group. The elementary fields $\Phi_{i}$ must then correspond to a set of primary scalars whose scaling dimensions are determined by the eigenvalues of $\Delta$. The general result [15] for the spectrum of the dilation operator in CFTs imposes then a further constrain on $\Delta$ : even though it is in general not symmetric it should be diagonalizable with real eigenvalues $\geq 1$.

\section{References}

[1] Z. Komargodski and A. Schwimmer, "On Renormalization Group Flows in Four Dimensions," JHEP 1112, 099 (2011) [arXiv:1107.3987 [hep-th]].

[2] Z. Komargodski, "The Constraints of Conformal Symmetry on RG Flows," arXiv:1112.4538 [hep-th].

[3] J. -F. Fortin, B. Grinstein and A. Stergiou, "Scale without Conformal Invariance in Four Dimensions," arXiv:1206.2921 [hep-th].

[4] J. -F. Fortin, B. Grinstein and A. Stergiou, to appear simultaneously with version 2 of the present work.

[5] J.-F. Fortin, B. Grinstein and A. Stergiou, "Scale without Conformal Invariance: An Example," Phys. Lett. B 704, 74 (2011) [arXiv:1106.2540 [hep-th]]; "Scale without Conformal Invariance: Theoretical Foundations," JHEP 1207, 025 (2012) [arXiv:1107.3840 [hep-th]];

"Scale without Conformal Invariance at Three Loops," JHEP 1208, 085 (2012) [arXiv:1202.4757 [hep-th]].

[6] J. -F. Fortin, B. Grinstein and A. Stergiou, "A generalized c-theorem and the consistency of scale without conformal invariance," arXiv:1208.3674 [hep-th].

[7] H. Osborn, "Derivation of a 4-dimensiona $c$ theorem," Phys. Lett. B 222, 97 (1989). 
[8] I. Jack and H. Osborn, "Analogs For The C Theorem For Four-dimensional Renormalizable Field Theories," Nucl. Phys. B 343, 647 (1990).

[9] H. Osborn, "Weyl consistency conditions and a local renormalization group equation for general renormalizable field theories," Nucl. Phys. B 363, 486 (1991).

[10] D. M. Capper and M. J. Duff, "Trace anomalies in dimensional regularization," Nuovo Cim. A 23, 173 (1974);

M. J. Duff, "Twenty years of the Weyl anomaly," Class. Quant. Grav. 11, 1387 (1994) [hep-th/9308075].

[11] A. Nicolis, R. Rattazzi and E. Trincherini, "The Galileon as a local modification of gravity," Phys. Rev. D 79, 064036 (2009) [arXiv:0811.2197 [hep-th]].

[12] M. B. Green and J. H. Schwarz, "Anomaly Cancellation in Supersymmetric D=10 Gauge Theory and Superstring Theory," Phys. Lett. B 149, 117 (1984).

[13] B. Keren-Zur, M. A. Luty and R. Rattazzi, work in progress.

[14] L. S. Brown, "Dimensional Regularization Of Composite Operators in Scalar Field Theory," Annals Phys. 126, 135 (1980).

[15] G. Mack, "All Unitary Ray Representations of the Conformal Group SU(2,2) with Positive Energy," Commun. Math. Phys. 55, 1 (1977).

[16] Y. Nakayama, "Supercurrent, Supervirial and Superimprovement," arXiv:1208.4726 [hep-th].

[17] J. Polchinski, "Scale And Conformal Invariance in Quantum Field Theory," Nucl. Phys. B 303, 226 (1988).

[18] A. B. Zamolodchikov, "Irreversibility of the Flux of the Renormalization Group in a 2D Field Theory," JETP Lett. 43, 730 (1986) [Pisma Zh. Eksp. Teor. Fiz. 43, 565 (1986)].

[19] C. G. Callan, Jr., S. R. Coleman and R. Jackiw, "A New improved energy momentum tensor," Annals Phys. 59, 42 (1970).

[20] Y. Nakayama, "On $\epsilon$-conjecture in a-theorem," arXiv:1110.2586 [hep-th].

[21] J. Wess, Nuovo Cim. 18, 1086 (1960).

[22] S. R. Coleman and R. Jackiw, "Why dilatation generators do not generate dilatations?," Annals Phys. 67, 552 (1971). 
[23] V. Riva and J. L. Cardy, Phys. Lett. B 622, 339 (2005) [hep-th/0504197].

[24] Y. Nakayama, "Scale invariance vs conformal invariance from holography," Int. J. Mod. Phys. A 25, 4849 (2010); "Gravity Dual for Cyclic Renormalization Group Flow without Conformal Invariance," arXiv:1107.2928 [hep-th]. 